\title{
Are Banks Too Big to Fail? Measuring Systemic Importance of Financial Institutions*
}

\begin{abstract}
Chen Zhou
De Nederlandsche Bank and Erasmus University Rotterdam

This paper considers three measures of the systemic importance of a financial institution within an interconnected financial system. The measures are applied to study the relation between the size of a financial institution and its systemic importance. Both the theoretical model and empirical analysis reveal that, when analyzing the systemic risk posed by one financial institution to the system, size should not be considered as a proxy of systemic importance. In other words, the "too big to fail" argument is not always valid, and measures of systemic importance should be considered. We provide the estimation methodology of systemic importance measures under the multivariate extreme value theory (EVT) framework.
\end{abstract}

JEL Codes: G21, C14.

\section{Introduction}

During financial crises, authorities have an incentive to prevent the failure of a financial institution because such a failure would pose a significant risk to the financial system, and consequently to the broader economy. A bailout is usually supported by the argument that a financial firm is "too big to fail": that is, larger banks exhibit higher systemic importance. A natural question arising from the debate on bailing out large financial firms is why particularly large

${ }^{*}$ The author thanks Markus Brunnermeier, Gabriele Galati, Douglas Gale, Miguel Segoviano, Stefan Straetmans, Casper de Vries, Haibin Zhu, participants at the second Financial Stability conference of the International Journal of Central Banking, and an anonymous referee for useful discussion and suggestions. Views expressed do not necessarily reflect official positions of De Nederlandsche Bank. Author postal address: Economics and Research Division, De Nederlandsche Bank, 1000AB Amsterdam, The Netherlands. E-mail: C.Zhou@DNB.nl. 
banks should be favored: are banks really too big to fail? An equivalent question might also be posed: does the size of a bank really matter for its systemic impact if it fails? The major difficulty in answering such a question is to design measures on the systemic importance of financial institutions. More specifically, we need to measure to what extent the failure of a particular bank will "contribute" to the systemic risk.

This paper deals with the problem in four steps. Firstly, we discuss the potential drawbacks of existing measures of systemic importance and propose alternatives that overcome these drawbacks. Secondly, we construct a theoretical model to assess whether larger banks correspond with higher systemic importance. Thirdly, we employ statistical methodology in estimating such measures within a constructed system consisting of twenty-eight U.S. banks. Finally, we use the estimated systemic importance measures and the size measures to empirically test the "too big to fail" statement.

Although the term "too big to fail" appears frequently in support of bailout activities, its downside is well acknowledged in the literature. Besides the distortion of the market discipline, the preference given to large financial firms encourages excessive risk-taking behavior, which potentially imposes more risk. Therefore, using the "too big to fail" argument to support intervention will result in a moral hazard problem: large firms that the government is compelled to support were among the greatest risk takers during the boom period. Furthermore, such a moral hazard problem will provide an incentive for firms to grow in order to be perceived as "too big to fail" (see Stern and Feldman 2004 for more discussions on the moral hazard problem).

Recently, both policymakers and academicians have begun to distinguish the size of a financial institution from the systemic importance it has by introducing new terms focusing on what the potential systemic impact might be if that particular institution fails. For example, Bernanke (2009) addresses the problem of financial institutions that are deemed "too interconnected to fail"; Rajan (2009) uses the term "too systemic to fail" to set the central focus of new regulation development. This urges the design of alternative measures on systemic importance. Measuring the systemic importance of financial institutions is particularly important for policymakers. It is the key issue in both financial stability assessment 
and macroprudential supervision. On the one hand, during crises, it is necessary to have such measures in order to justify bailout actions. On the other hand, it is crucial to supervise and monitor banks with higher systemic importance during regular periods. Policy proposals for stabilizing the financial system always rely on such measures. For instance, charging a deposit insurance premium is an alternative proposed by Acharya, Santos, and Yorulmazer (2010) for defending systemic risk. Systemic importance measures can serve as an indicator for pricing the corresponding insurance premium or taxation.

A few applicable measures of systemic importance have appeared in recent empirical studies. Adrian and Brunnermeier (2008) proposed the conditional value-at-risk $(\mathrm{CoVaR})$ for measuring risk spillover. Similar to the value-at-risk measure, which quantifies the unconditional tail risk of a financial institution, the CoVaR quantifies how the financial stress of one institution can increase the tail risk of others. These measures demonstrate the bilateral relation between the tail risks of two financial institutions. The setup of the CoVaR measure indicates that it is designed for bilateral risk spillover. When applying CoVaR to assess the systemic importance of one financial institution to the system, it is necessary to construct a system indicator on the status of the system and then to analyze the bilateral relation between the system indicator and a specific bank. However, the complexity of the financial system is of a higher order than bilateral relations. Thus, a general indicator of the system is usually difficult to construct. Furthermore, the CoVaR measure is difficult to generalize into a systemic context in order to analyze multiple financial institutions all together. As an alternative, Segoviano and Goodhart (2009) introduced the "probability that at least one bank becomes distressed" (PAO). Comparing these two measures, we observe that the CoVaR is a measure of conditional quantile, while the PAO is a measure of conditional probability, which acts as the counterpart of conditional quantile. Within a probability setup, generalization from bivariate to multivariate is possible. However, the PAO measure focuses on the probability of having a systemic impact that there will be at least one extra crisis, without specifying how severe the systemic impact is. Therefore, the measure may not provide sufficient information on the systemic importance of a financial institution. Our empirical results partially reflect the 
less informative feature of the PAO: the PAO measures remain at a constant level across different financial institutions and across time.

Extending the PAO measure while staying within the multivariate context, we propose the systemic impact index (SII), which measures the expected number of bank failures in the banking system given a situation in which one particular bank fails. Clearly, the SII measure emphasizes more the systemic impact. We also consider a reversed measure: the probability that a particular bank fails, given that there exists at least one other failure in the system. This we refer to as the vulnerability index (VI).

To test "too big to fail," we first consider a theoretical model from which both size and systemic importance measures can be explicitly calculated. The model stems from the literature on systemic risk. In the literature, two categories of models consider crisis contagion and systemic risk in the banking system: banks are systemically linked via either direct channels such as interbank markets or indirect channels such as similar portfolio holdings in bank balance sheets. Studies in the first category focus on the contagion effect; i.e., a crisis in one financial institution may cause crises in the others. The second category of studies focuses on modeling systemic risk; i.e., crises of different financial institutions may occur simultaneously. For the first category of studies, particularly on modeling the interbank market, see Allen and Gale (2000), Freixas, Parigi, and Rochet (2000), and Dasgupta (2004). Cifuentes, Shin, and Ferrucci (2005) consider two channels: similar portfolio holdings and mutual credit exposure. They show that contagion is mainly driven by changes in asset prices. Hence the indirect channel dominates. For models focusing on the indirect channel, Lagunoff and Schreft (2001) assume that the return of one bank's portfolio depends on the portfolio allocation of other banks, and they show that crises can either spread from one bank to another or happen simultaneously due to forward-looking behavior. De Vries (2005) starts from the fat-tail property of the underlying assets shared by banks, and he argues that this creates the potential for systemic breakdown. For an overview of contagion and systemic risk modeling, we refer to de Bandt and Hartmann (2001) and Allen, Carletti, and Babus (2009).

The contagion literature focuses mainly on explaining the existence of a contagion effect: how a crisis in one financial institution 
leads to a crisis in another. Thus, the models usually consider the risk spillover between only two banks. To address the financial system as a complex entity, several studies have considered network models combined with bilateral spillover. Following those theoretical studies, empirical analyses, such as the CoVaR measure, were then designed for measuring bilateral relations. In order to address the systemic importance issue within a systemic context, we must consider a multibank approach. Thus, we establish our theoretical model based on the indirect channel models: similar portfolio holdings lead to the possibility of simultaneous crises. The fundamental intuition is that banks are interconnected due to the common exposures on their balance sheets. Thus, the systemic importance of a particular bank is closely associated with the number of different risky banking activities in which the bank participates. This, in turn, may not be directly associated with the bank's size. In other words, "too big to fail" is not always valid. Our model further shows that banks concentrating on few specific activities can grow large without increasing their systemic importance.

We acknowledge a potential downside of considering the indirect channel model: it does not provide a model on the causality effect. Nevertheless, even without addressing the contagion effect, investigating systemic importance based on the systemic risk of having simultaneous crises is an important issue. This comes from the "too many to fail" phenomenon discussed by Acharya and Yorulmazer (2007). They consider a game theory approach and show that because regulators would bail out a bank in distress only if a large part of the system suffers from distresses, individual banks would have an incentive to hold similar portfolios in order to increase the possibility of being rescued when a crisis occurs. Such a "too many to fail" phenomenon is in line with the intuition that when evaluating systemic importance of a financial institution, it is necessary to evaluate to what extent the crisis of the financial institution is accompanied by crises of others. Therefore, we choose to build the systemic importance measure on an indirect channel model.

The theoretical finding that "too big to fail" is not always valid contributes to policy discussions on micro-level risk management and macro-level banking supervision. Since diversification is the usual tool in micro-level risk control, financial institutions, particularly the large ones, tend to take part in more banking activities in order 
to diversify away their individual risk. This may, however, increase their systemic importance. It is important to acknowledge the tradeoff between managing individual risk and maintaining independence within the entire banking system. Portfolio construction toward reduction in individual risk may imply a transfer of risk to systemic linkage and thus increase the systemic importance. Therefore, prudential regulations which limit individual risk taking, such as Basel I and II type regulations, are not sufficient for maintaining stability of the entire financial system. Macroprudential supervision which considers the system as a whole is necessary for achieving financial stability. A macroprudential approach requires careful consideration of both individual risk taking and the systemic importance of each individual financial institution.

Next, we demonstrate how to empirically estimate the proposed systemic importance measures. We adopt the multivariate extreme value theory (EVT) framework for empirical estimation. In any investigation of crises, or rare events, the major difficulty is the scarcity of observations on crisis events. Since our intention is to address the interconnectedness of the banking crises, which is in effect a joint crisis, the difficulty with regard to the shortfall in observations is further enhanced. A modern statistical instrumentEVT - fills the gap. The essential idea of EVT is to model the intermediate-level observations, which are close to extreme, and extrapolate the observed properties into an extreme level. Therefore, the interconnectedness of crises can be approximated by the interconnectedness of tail events, which are not necessarily at a crisis level. Univariate EVT has been applied in value-at-risk assessment for individual risks (see, e.g., Embrechts, Klüppelberg, and Mikosch 1997). Recent developments on multivariate EVT provide the opportunity to investigate extreme co-movements, which serves our purpose. For instance, EVT was applied to measure risk contagions across different financial markets in Hartmann, Straetmans, and de Vries (2004) and Poon, Rockinger, and Tawn (2004). An application of multivariate EVT in analyzing bilateral relations within the banking system can be found in Hartmann, Straetmans, and de Vries (2005). Beyond bivariate relations, the Global Financial Stability Report published by the International Monetary Fund in April 2009 (IMF 2009) demonstrates - using EVT analysis - the interconnection of financial distress within a system consisting of three banks. 
Our empirical estimation of the systemic importance measures uses multivariate EVT without restricting the number of banks under consideration.

We provide an empirical methodology with which to estimate the systemic importance measures-PAO, SII, and VI-under the multivariate EVT framework. We conduct an exercise for a constructed system consisting of twenty-eight U.S. banks and test the correlation between the systemic importance measures and different measures on size. We find that, in general, systemic importance measures are not correlated with all bank size measures. Hence, the size of a financial institution should not be considered as a proxy of its systemic importance without careful justification. This agrees with our theoretical model. Overall, we conclude that it is necessary to have proper systemic importance measures for identifying the systemically important financial institutions.

\section{Systemic Importance Measures}

We consider a banking system containing $d$ banks with their status indicated by $\left(X_{1}, \ldots, X_{d}\right)$, where an extremely high value of $X_{i}$ indicates a distress situation or a crisis in bank $i$. Potential candidates for such an indicator might include the loss of equity returns, loss returns on total asset, or credit default swap (CDS) rates.

To define a crisis, it is necessary to consider what constitutes a properly high threshold. Our approach takes value-at-risk as such a threshold. For the distress status $X$ of a financial institution, a VaR at a tail probability level $p$ is defined by

$$
P(X>\operatorname{VaR}(p))=p .
$$

Prudential regulations consider the $p$-level as 1 percent or 0.1 percent in order to evaluate risk-taking behavior of an individual institution. We say that a bank is in crisis if $X>\operatorname{VaR}(p)$ with an extremely low $p$. Here, we do not specify the level $p$ explicitly. Instead, we impose a restriction that the $p$-level in the definition of banking crises is constant across all banks. Notice that banks may differ in their risk profiles, which results in different endurability on risk, i.e., different $\operatorname{VaR}(p)$. Thus, a unified level of loss for crisis definition may not fit the diversified situation of different financial institutions. Instead, 
an extreme event $X>\operatorname{VaR}(p)$ corresponds to a return frequency as $1 / p$. Fixing such a frequency for crisis definition takes account of the diversity of bank risk profiles. Furthermore, such a definition is aligned with the usual crisis description: for example, with yearly data, a $p$ equal to $1 / 50$ corresponds to "a crisis once per fifty years."

The systemic importance measures consider the impact on other financial institutions when one of them falls into crisis. We start from the measure proposed by Segoviano and Goodhart (2009): the conditional probability of having at least one extra bank failure, given that a particular bank fails (PAO). In our model, this measure is the following probability:

$$
P A O_{i}(p):=P\left(\left\{\exists j \neq i \text {, s.t. } X_{j}>\operatorname{VaR}_{j}(p)\right\} \mid X_{i}>\operatorname{VaR}(p)\right) .
$$

We argue that the PAO measure may not provide sufficient information for identifying the systemically important banks. Consider the following example. Suppose we have a banking system with banks categorized into two separate groups. Banks within each group are strongly linked, while banks from the two groups are independent of each other. One group contains only two banks, $X_{1}$ and $X_{2}$, while the other group contains more banks, $X_{3}, \ldots, X_{d}, d>4$. In other words, $X_{1}$ and $X_{2}$ are highly related; $X_{3}, \ldots, X_{d}$ are highly related; and $X_{i}$ and $X_{j}$ are independent for any $1 \leq i \leq 2$ and $3 \leq j \leq d$.

Then the PAO measure for $X_{1}, P A O_{1}$, will be close to 1 since a crisis of $X_{1}$ will be accompanied by a crisis of $X_{2}$. However, the PAO measure for $\mathrm{X}_{3}, \mathrm{PAO}_{3}$, will also be close to 1 because of similar reasoning. When $d$ is high, it is clear that $X_{3}$ is more systemically important than $X_{1}$ because it is associated with a larger fraction of the entire banking system. However, this will not be reflected by the comparison between $P A O_{1}$ and $\mathrm{PAO}_{3}$. In this example, $P A O_{1}$ and $\mathrm{PAO}_{3}$ should be at a high, comparable level.

Generally speaking, the PAO measure only provides the probability of a systemic impact when one particular bank fails - that is, an extra crisis occurring in other financial institutions. It does not specify the size of such an impact - that is, the number of extra crises in the entire system. Hence, if every institution in the system is connected to a certain fraction of the system, the PAO measures 
of all should stay at a high, comparable level. With indistinguishable PAO measures, it is not sufficient to identify the systemically important financial institutions.

A natural extension of the PAO measure is to consider the expected number of failures in the system, given that a particular bank fails. This is defined as our systemic impact index (SII). Using the notation above, it can be written as

$$
\operatorname{SII}_{i}(p):=E\left(\sum_{j=1}^{d} 1_{X_{j}>\operatorname{VaR}(p)} \mid X_{i}>\operatorname{VaR}_{i}(p)\right),
$$

where $1_{A}$ is the indicator function that is equal to 1 when $A$ holds, and is 0 otherwise.

Since the PAO and SII measures characterize the outlook of the financial system when a particular bank fails, a reverse question is what the probability of a particular bank failure is when the system exhibits some distress. To characterize the system distress, we use the same term as in the PAO measure: there exists at least one other bank failure. Hence, we define a vulnerability index (VI) by swapping the two items in the PAO definition as follows:

$$
V I_{i}(p):=P\left(X_{i}>\operatorname{VaR}(p) \mid\left\{\exists j \neq i \text {, s.t. } X_{j}>\operatorname{VaR}(p)\right\}\right) .
$$

From the definitions, all three measures summarize specific information on the risk spillover in the banking system. It is necessary to consider all of them when assessing the systemic importance of financial institutions.

\section{Extreme Value Theory and Systemic Importance Measures}

\subsection{The Setup of Extreme Value Theory}

Consider our $d$-bank setup. Modeling the crisis of a particular financial institution $i$ corresponds to modeling the tail distribution of $X_{i}$. Moreover, modeling the systemic risk-i.e., the extreme comovements among $\left(X_{1}, \ldots, X_{d}\right)$-corresponds to modeling the tail dependence structure of $\left(X_{1}, \ldots, X_{d}\right)$. Extreme value theory provides models for such a purpose. 
To assess VaR with a low probability level $p$, univariate EVT can be applied in modeling the tail behavior of the loss. Since our focus is on systemic risk, we omit the details on univariate risk modeling (see, e.g., Embrechts, Küppelberg, and Mikosch 1997). Multivariate EVT models consider not only the tail behavior of individual $X_{i}$ but also the extreme co-movements among them.

The fundamental setup of multivariate EVT is as follows. For any $x_{1}, x_{2}, \ldots, x_{d}>0$, as $p \rightarrow 0$, we assume that

$$
\begin{aligned}
& \frac{P\left(X_{1}>\operatorname{VaR}_{1}\left(x_{1} p\right) \text { or } \cdots \text { or } X_{d}>\operatorname{VaR}_{d}\left(x_{d} p\right)\right)}{p} \\
& \quad \rightarrow L\left(x_{1}, x_{2}, \ldots, x_{d}\right),
\end{aligned}
$$

where $V a R_{i}$ denotes the value-at-risk of $X_{i}$, and $L$ is a finite positive function. ${ }^{1}$ The $L$ function characterizes the co-movement of extreme events that $X_{i}$ exceeds a high threshold $\operatorname{VaR}_{i}\left(x_{i} p\right) .\left(x_{1}, \ldots, x_{d}\right)$ controls the level of high threshold, which in turn controls the direction of extreme co-movement. For the property on the $L$ function, see de Haan and Ferreira (2006).

The value of $L$ at a specific point, $L(1,1, \ldots, 1)$, is a measure of the systemic linkage of banking crises among the $d$ banks. From the definition in (4), we have

$$
L(1,1, \ldots, 1)=\lim _{p \rightarrow 0} \frac{P\left(X_{1}>\operatorname{VaR} R_{1}(p) \text { or } \ldots \text { or } X_{d}>\operatorname{VaR}(p)\right)}{p} .
$$

In the context of our banking system, when $p$ is at a low level, it approximates the quotient ratio between the probability that there exists at least one bank in crisis and the tail probability $p$ used in the definition of individual crisis. For the bivariate case, this was considered by Hartmann, Straetmans, and de Vries (2004) in measuring systemic risk across different financial markets.

\footnotetext{
${ }^{1}$ Notice that considering the union of the events-i.e., using "or" in (4) - is simply a result of the definition of the distribution function. Define $F\left(x_{1}, \ldots, x_{d}\right)=P\left(X_{1} \leq x_{1}, \ldots, X_{d} \leq x_{d}\right)$ as the distribution function of $\left(X_{1}, \ldots, X_{d}\right)$. In order to consider the tail property, the assumption is made on the tail part $1-F$, which is the probability of the union of extreme events as in relation (4).
} 
Note that the $L$ function is connected with the modern instrument of dependence modeling - the copula. Denote the joint distribution function of $\left(X_{1}, \ldots, X_{d}\right)$ as $F\left(x_{1}, \ldots, x_{d}\right)$ while the marginal distributions are denoted as $F_{i}\left(x_{i}\right)$ for $i=1, \ldots, d$. Then there exists a unique distribution function $C\left(x_{1}, \ldots, x_{d}\right)$ on $[0,1]^{d}$, such that

$$
F\left(x_{1}, \ldots, x_{d}\right)=C\left(F_{1}\left(x_{1}\right), \ldots, F_{d}\left(x_{d}\right)\right),
$$

where all marginal distributions of $C$ are standard uniform distributions. $C$ is called the copula. By decomposing $F$ into marginal distributions and copula, we separate the marginal information from the dependence structure summarized in the copula $C$. Condition (4) is equivalent to the following relation. For any $x_{1}, x_{2}, \ldots, x_{d}>0$, as $p \rightarrow 0$,

$$
\frac{1-C\left(1-p x_{1}, \ldots, 1-p x_{d}\right)}{p} \rightarrow L\left(x_{1}, x_{2}, \ldots, x_{d}\right) .
$$

Hence the $L$ function characterizes the limit behavior of the copula $C$ at the corner point $(1, \ldots, 1) \in[0,1]^{d}$. In other words, the $L$ function captures the tail behavior of the copula $C$.

Linking the $L$ function to the tail behavior of copula yields the two following views. Firstly, since it is connected to the copula, the $L$ function does not contain any marginal information. Thus, in modeling the linkage of banking crises, the $L$ function is irrelevant to the risk profile of the individual bank. Secondly, in characterizing the tail behavior of a copula, the $L$ function does not contain dependence information at a moderate level, as in the copula $C$. Instead, $L$ only contains tail dependence information. To summarize, the $L$ function contains the minimal amount of required information in modeling extreme co-movements. Therefore, models on $L$ are flexible to accommodate all potential marginal risk profiles and potential moderate-level dependence structures. Compared to Segoviano and Goodhart (2009), who consider the Consistent Information Multivariate Density Optimizing (CIMDO) approach on estimating the copula $C$, since models on the copula $C$ incorporate the interconnection of banking systems in regular time, estimating a copula model may misspecify the tail dependence structure. Because we intend to model the interconnection of banking crises, considering 
the $L$ function in the multivariate EVT approach is sufficient and less restrictive.

\subsection{Systemic Importance Measures under Multivariate EVT}

Under the multivariate EVT setup, the limit of the three systemic importance measures can be directly calculated from the $L$ function. Notice that in the definitions of these measures, the probability level $p$ for defining crisis is considered. However, we prove that, as $p \rightarrow 0$, the systemic importance measures can be well approximated by their limits.

The following proposition shows the limit of the PAO measure. The proof is in appendix 1 .

Proposition 1. Suppose $\left(X_{1}, X_{2}, \ldots X_{d}\right)$ follows the multivariate EVT setup. With the definition of PAO in (1), we have

$$
P A O_{i}:=\lim _{p \rightarrow 0} P A O_{i}(p)=L_{\neq i}(1,1, \ldots, 1)+1-L(1,1, \ldots, 1),
$$

where $L$ is the $L$ function characterizing the tail dependence of $\left(X_{1}, \ldots, X_{d}\right)$, and $L_{\neq i}(1,1, \ldots, 1)$ is the $L$ function characterizing the tail dependence of $\left(X_{1}, \ldots, X_{i-1}, X_{i+1}, \ldots X_{d}\right)$.

Notice that $L$ is defined on $\mathbb{R}^{d}$, while $L_{\neq i}$ is defined on $\mathbb{R}^{d-1}$. Moreover,

$$
L_{\neq i}(1,1, \ldots, 1)=L(1,1, \ldots, 1,0,1, \ldots, 1),
$$

where 0 appears at the $i$-th dimension.

Proposition 1 shows that when considering a low-level $p$, the measure $P A O_{i}(p)$ is close to its limit denoted by $P A O_{i}$. For calculating $P A O_{i}$, it is sufficient if the $L$ function is known. Therefore, we could have a proxy of the PAO measure with low-level $p$ by estimating the $L$ function. In a theoretical model, the $L$ function can be explicitly calculated. For an empirical analysis, the $L$ function can be estimated from historical data. We provide a practical guide for estimating the $L$ function in appendix 2. For more discussions, see de Haan and Ferreira (2006).

Analogous to that of $P A O$, the limit of $V I(p)$ exists under the multivariate EVT setup. We present the result in the following proposition but omit the proof. 
Proposition 2. Suppose $\left(X_{1}, X_{2}, \ldots X_{d}\right)$ follows the multivariate EVT setup. With the definition of VI in (3), we have

$$
V I_{i}:=\lim _{p \rightarrow 0} V I_{i}(p)=\frac{L_{\neq i}(1,1, \ldots, 1)+1-L(1,1, \ldots, 1)}{L_{\neq i}(1,1, \ldots, 1)},
$$

with the same notation defined in proposition 1.

From propositions 1 and 2, we get the following corollary.

Corollary 1. PAO $>P A O_{j}$ holds if and only if $V I_{i}>V I_{j}$.

Corollary 1 implies that when considering the ranking instead of the absolute level, the VI measure is in fact as informative as the PAO measure.

The following proposition shows how to calculate the limit of SII under the multivariate EVT setup. The proof appears in appendix 1.

Proposition 3. Suppose $\left(X_{1}, X_{2}, \ldots X_{d}\right)$ follows the multivariate EVT setup. With the definition of SII in (2), we have

$$
S I I_{i}:=\lim _{p \rightarrow 0} S I I_{i}(p)=\sum_{j=1}^{d}\left(2-L_{i, j}(1,1)\right),
$$

where $L_{i, j}$ is the $L$ function characterizing the tail dependence of $\left(X_{i}, X_{j}\right)$.

Notice that

$$
L_{i, j}(1,1)=L(0, \ldots, 0,1,0, \ldots, 0,1,0, \ldots, 0),
$$

where 1 appears only at the $i$-th and $j$-th dimensions. We remark that $2-L_{i, j}(1,1)$ is in fact a measure of bilateral relation between the crises of banks $X_{i}$ and $X_{j}$. Thus, the SII measure is an aggregation of measures on bilateral relations. This is parallel to the spillover index studied in Diebold and Yilmaz (2009) when measuring volatility spillover in a multivariate system: after measuring the volatility spillover between each pair, the spillover index is an aggregation of the measures on bilateral relations. 
Again, proposition 3 shows that $S I I_{i}$ is a good approximation of $S I I_{i}(p)$ when $p$ is at a low level. And the estimation of $S I I_{i}$ is only based on the estimation of the $L$ function. From the calculation of PAO and SII, it is clear that the two measures provide different information on systemic importance. A ranking based on PAO does not necessarily imply the same ranking on SII. Thus, it is still necessary to look at both of the measures in order to obtain a complete picture on the systemic importance of a bank.

To summarize, the multivariate EVT setup provides the opportunity to evaluate all three systemic importance measures when the $L$ function is known. Since the $L$ function characterizes the tail dependence structure in $\left(X_{1}, \ldots, X_{d}\right)$, all the systemic importance measures can be viewed as characterizations of the tail dependence among banking crises.

\section{Are Banks "Too Big to Fail"? A Theoretical Model}

We construct a simple model showing that large banks might have a lower level of systemic importance compared with small banks: banks are not necessarily too big to fail.

We start by reviewing a simple model in de Vries (2005), which explains the systemic risk within a two-bank system.

Consider two banks $\left(X_{1}, X_{2}\right)$ holding exposures on two independent projects $\left(Y_{1}, Y_{2}\right)$, as in the following affine portfolio model:

$$
\left\{\begin{array}{l}
X_{1}=(1-\gamma) Y_{1}+\gamma Y_{2} \\
X_{2}=\gamma Y_{1}+(1-\gamma) Y_{2}
\end{array}\right.
$$

where $0<\gamma<1,\left(Y_{1}, Y_{2}\right)$ indicates the loss returns of the two projects. To measure the systemic risk, de Vries (2005) considers the following measure:

$$
\lim _{s \rightarrow \infty} E(\kappa \mid \kappa \geq 1):=\lim _{s \rightarrow \infty} \frac{P\left(X_{1}>s\right)+P\left(X_{2}>s\right)}{P\left(X_{1}>s \text { or } X_{2}>s\right)} .
$$

Intuitively, $E(\kappa \mid \kappa \geq 1)$ is the expected number of bank crises in the two-bank system, given that at least one bank is in crisis. Here, the crisis of $X_{i}$ is defined as $X_{i}>s$. It is proved that when $Y_{i}, i=1,2$ are normally distributed, $\lim _{s \rightarrow \infty} E(\kappa \mid \kappa \geq 1)=1$. Thus, given that there exists at least one bank in crisis, the expected total number 
of crises is 1 . Hence, there is no extra crisis except the existing one. This is called a weak fragility case. In other words, the systemic impact does not exist. To the contrary, suppose $Y_{i}, i=1,2$ follow a heavy-tailed distribution on the right tail. The result differs. The heavy-tailed distribution is defined as

$$
\left\{\begin{aligned}
P\left(Y_{i}>s\right) & =s^{-\alpha} K(s), i=1,2, \\
P\left(Y_{i}<-s\right) & =o\left(P\left(Y_{i}>s\right)\right),
\end{aligned}\right.
$$

where $\alpha>0$ is called the tail index and $K(s)$ is a slowly varying function satisfying

$$
\lim _{t \rightarrow \infty} \frac{K(t s)}{K(s)}=1,
$$

for all $s>0$. De Vries (2005) proved that for $\gamma \in[1 / 2,1]$,

$$
\lim _{s \rightarrow \infty} E(\kappa \mid \kappa \geq 1)=1+(1 / \gamma-1)^{\alpha}>1 .
$$

This is called the strong fragility case because one existing crisis will be accompanied by potential extra crises. The empirical literature has extensively documented that the losses of asset returns follow heavy-tailed distributions. Therefore, the latter model based on heavy-tailed distributions reflects the empirical observations and explains the systemic risk existing in the financial system.

We remark that when assuming the heavy-tailedness of $\left(Y_{1}, Y_{2}\right)$, and the affine portfolio model in (9), it is a direct consequence that $\left(X_{1}, X_{2}\right)$ follows a two-dimensional EVT setup. ${ }^{2}$ Moreover, if $Y_{1}$ and $Y_{2}$ are identically distributed, for a fixed tail probability $p$, the VaRs of $X_{1}$ and $X_{2}$ are equal; i.e., $\operatorname{VaR}_{1}(p)=V a R_{2}(p)$. Replacing $s$ with $\operatorname{VaR}_{i}(p)$ in the definition of the systemic risk measure (10), and asking $p \rightarrow 0$, we get

$$
\begin{aligned}
\lim _{p \rightarrow 0} E(\kappa \mid \kappa \geq 1) & :=\lim _{p \rightarrow 0} \frac{P\left(X_{1}>\operatorname{VaR}(p)\right)+P\left(X_{2}>\operatorname{VaR}(p)\right)}{P\left(X_{1}>\operatorname{VaR}(p) \text { or } X_{2}>\operatorname{VaR}(p)\right)} \\
& =\frac{2}{L(1,1)} .
\end{aligned}
$$

\footnotetext{
${ }^{2}$ For a formal proof, see Zhou (2008, ch. 5).
} 
Therefore, the setup in de Vries (2005) imposes a multivariate EVT setup, and the measure on the systemic risk is essentially based on $L(1,1)$.

We point out that within this two-bank, two-project model, it is not possible to differentiate the systemic importance of the two banks. From the model and from proposition 1, we get

$$
S I I_{i}=3-L(1,1), \quad i=1,2 .
$$

Hence the two banks have equal systemic importance measured by SII. Similar results hold for the other two measures, PAO and VI. Intuitively, within a two-bank setup, the linkage of crises is a mutual bilateral relation. Hence, one could not distinguish the systemic importance of the two banks. In order to construct a model in which it is possible to compare the systemic importance at different levels, it would be necessary to generalize the de Vries (2005) model to a system consisting of at least three banks.

Next, we consider the size issue. In the de Vries (2005) two-bank model, suppose that both of the two banks have total capital 1, and both of the two projects receive capital 1. According to the affine portfolio model (9), the capital market is clear. In this case, the two banks have the same size in terms of total assets. In order to differentiate the sizes of the banks, a more complex affine portfolio model is necessary.

Addressing the two above-mentioned points, we consider a model with three banks $\left(X_{1}, X_{2}, X_{3}\right)$ and three independent projects $\left(Y_{1}, Y_{2}, Y_{3}\right)$. Suppose $X_{1}$ holds capital 2 for investment, while $X_{2}$ and $X_{3}$ hold capital 1 each. Moreover, suppose the project $Y_{1}$ demands an investment 2, while $Y_{2}$ and $Y_{3}$ each have a capital demand 1. Then the market is clear, with the following affine portfolio model:

$$
\left\{\begin{array}{l}
X_{1}=(2-2 \gamma) Y_{1}+\gamma Y_{2}+\gamma Y_{3} \\
X_{2}=\gamma Y_{1}+(1-\gamma-\mu) Y_{2}+\mu Y_{3}, \\
X_{3}=\gamma Y_{1}+\mu Y_{2}+(1-\gamma-\mu) Y_{3},
\end{array}\right.
$$

where $0<\gamma, \mu<1$, and $\gamma+\mu<1$. Clearly, this is not the only possible allocation for market clearance. Nevertheless, it is sufficient to demonstrate our argument regarding the "too big to fail" problem. Notice that $X_{1}$ is a larger bank compared with $X_{2}$ and $X_{3}$. Here, the 
size refers to the total investment in the risky projects. We intend to compare the systemic importance of $X_{1}$ with that of $X_{2}$ and $X_{3}$.

The two parameters $\gamma$ and $\mu$ are interpreted as the control of similarity in portfolio holdings across the three banks. The parameter $\gamma$ controls the similarity between the large bank and the small banks. When $\gamma$ is close to 1 , the strategy of the large bank is different from that of the two small banks, while the two small banks hold similar portfolios. When $\gamma=1 / 2$, the large bank has exposures on all three projects proportional to their capital demands. Hence, the large bank is involved in all projects. When $\gamma$ is close to 0 , the large bank is again different from the two small banks. In the latest case, the similarity of the two small banks is further controlled by the parameter $\mu$ : a $\mu$ lying in the middle of $(0,1-\gamma)$ shows that the two small banks are similar in portfolio holding, while a $\mu$ lying close to the two corners of $(0,1-\gamma)$ corresponds to different strategies between the two small banks.

Suppose all $Y_{i}$ follow a heavy-tailed distribution defined in (11) for $i=1,2,3$. Then, similar to the two-bank case, $\left(X_{1}, X_{2}, X_{3}\right)$ follows a three-dimensional EVT setup. Instead of discussing all possible values on the parameters $(\gamma, \mu)$, we focus on three cases: $\gamma$ is close to $1, \gamma=1 / 2$, and $\gamma$ is close to 0 . The results from comparing the SII measures are in the following theorem. The proof is again in appendix 1.

ThEOREM 1. Consider a three-bank, three-project model with the affine portfolio given in (12). Suppose the losses of the three projects exhibit the same heavy-tailed feature as in (11), with $\alpha>1$. We have the following relations.

CASE 1: $\quad \frac{2}{3} \leq \gamma<1$

$$
S S I_{1}<1=S S I_{2}=S S I_{3} .
$$

CASE 2: $\quad \gamma=\frac{1}{2}$

$$
S S I_{1} \geq S S I_{2}=S S I_{3}
$$

The equality holds if and only if $\mu=1 / 4$.

CASE 3: $0<\gamma<\frac{1}{3}$ 
There exists a $\mu^{*}<\frac{1-\gamma}{2}$ such that for any $\mu$ satisfying $\mu^{*}<\mu<$ $1-\gamma-\mu^{*}$

$$
S S I_{1}<S S I_{2}=S S I_{3}
$$

On the other hand, for any $\mu$ satisfying $0<\mu<\mu^{*}$ or $1-\gamma-\mu^{*}<$ $\mu<1-\gamma$, we have

$$
S S I_{1}>S S I_{2}=S S I_{3} .
$$

When $\mu=\mu^{*}$ or $\mu=1-\gamma-\mu^{*}$,

$$
S I I_{1}=S I I_{2}=S I I_{3}
$$

The following lemma shows that the comparison among the PAO measures follows the comparison among the SII measures in the three-bank model.

LEMMA 1. With the assumptions in theorem 1, the order of PAO follows the order of SII; i.e., for any $i \neq j, P A O_{i}>P A O_{j}$ holds if and only if $S I I_{i}>S I I_{j}$.

Combining lemma 1 and corollary 1, we see that the order of VI also follows the order of SII in the simple three-bank model. Notice that the three-bank model is a very specific and simple case. The result in lemma 1 does not hold in a general context when the number of banks is more than three. Therefore, for empirical study within a multibank system, it is still necessary to estimate all three measures, which may provide different views.

We interpret the results in theorem 1 as follows.

If $\gamma$ is close to 1 , the large bank $X_{1}$ focuses on the two smaller projects $Y_{2}$ and $Y_{3}$, while small banks $X_{2}$ and $X_{3}$ focus on the large project $Y_{1}$. In this case, the balance sheet of the large bank is quite different from that of the small banks, while the two small banks are holding similar portfolios. Therefore, the large bank has less systemic linkage to the other two small banks. We observe that the large bank is less systemically important compared with the others; i.e., the large bank is not "too big to fail."

If $\gamma=1 / 2$, the large bank $X_{1}$ invests $(1,1 / 2,1 / 2)$ in three projects. Hence it is involved in all three projects, which creates 
the linkage to the other two small banks. In this case, it is "too big to fail." The inequality becomes an equation if and only if $\mu=1 / 4$. For $\mu=1 / 4$, the three banks all invest in three projects proportional to their capital demands. They have exactly the same strategy in managing their portfolios. A crisis in any of the three banks will be accompanied by crises in the other two. Therefore, they are equally systemically important. Excluding $\mu=1 / 4$, the large bank will be the most systemically important bank.

If $\gamma$ is close to 0 , the large bank $X_{1}$ focuses on the large project $Y_{1}$, while it still has exposures on $Y_{2}$ and $Y_{3}$. The small banks $X_{2}$ and $X_{3}$ focus on the two small projects $Y_{2}$ and $Y_{3}$. Now it matters how similar their portfolios are. If $\mu$ is in the middle $\left(\mu^{*}<\mu<1-\gamma-\mu^{*}\right)$, then the balance sheet composition of two small banks is relatively similar. Hence, they are more systemically important compared with the large bank. If $\mu$ is close to the corner $\left(0<\mu<\mu^{*}\right.$ or $1-\gamma-\mu^{*}<\mu<1-\gamma$ ), then the two small banks differ in their balance sheets. Since the large bank still has exposures on $Y_{2}$ and $Y_{3}$ equally, it is the most systemically important bank. It is worth mentioning that the systemic importance of bank $X_{1}$ is determined not only by its own risk positions but also by the risk-taking behavior of the others. Even though the portfolio of bank $X_{1}$ is fixed by fixing $\gamma$, the change of the portfolios hold by the other two banks can still result in a change of the systemic importance of $X_{1}$.

To summarize, we observe that "too big" is not necessarily the reason for being "too systemically important." Instead, a bank having a balance sheet that is exposed to more risky projects would cause it to become more systemically important. Here, we regard $Y_{i}, i=1,2,3$ as different risky projects. One may also regard them as different risky banking activities. Therefore, a bank that is more diversified in banking activities may turn out to be "too big to fail."

Notice that having a diversified balance sheet is the usual way of managing individual risk. In order to obtain the diversification, banks, particularly large banks, will be spurred on to take part in more banking activities. The above discussion shows that this will simultaneously result in a "too big to fail" problem. Conversely, a large bank specialized in a limited number of banking activities might be risky as an individual but at the same time less systemically important. There is a trade-off between managing individual risk and keeping a sense of independence within the entire banking 
system. For maintaining the stability of the financial system, it is necessary for the regulators to recognize such a trade-off and impose proper regulations in order to give banks incentives to balance their individual risk position and systemic importance.

\section{Empirical Results}

\subsection{Empirical Setup and Data}

We apply the three proposed measures of systemic importance to an artificially constructed financial system consisting of twenty-eight U.S. banks. After estimating the three measures, we calculate the correlation coefficients between these measures and the measures of the size of the banks. From the test on correlation coefficients, we can empirically test whether larger banks exhibit larger systemic importance, thereby testing the "too big to fail" argument. We also consider a moving window approach, which demonstrates the variation of the systemic importance measures across time.

The data set for constructing the systemic importance measures consists of daily equity returns of twenty-eight U.S. banks listed on the New York Stock Exchange (NYSE) from 1987 to 2009 (twenty-three years). ${ }^{3}$ The chosen banks are listed in table 1 with the descriptive statistics on their stock returns.

Regarding the size of the banks, the data set consists of various measures. We consider total assets, total equity, and total debt for the twenty-eight banks. ${ }^{4}$ The data that appear are reported in a yearly frequency from 1987 to 2009. For each bank, we present the end-of-2009 values as well as the average values across the twentythree years in table 2 .

From the descriptive statistics of the equity returns, we observe that all daily returns exhibit high kurtosis compared with the

\footnotetext{
${ }^{3}$ The data are obtained from Datastream. Three selection criteria are applied: the financial institutions should be classified in the sector "Banks"; they should be traded primarily on the NYSE (DS code starting with "U:"); and the time series should be active from the beginning of 1987 till the end of 2009. The selection procedure results in twenty-eight banks.

${ }^{4}$ The data on total assets, total equity, and total debt are obtained from Datastream, with item code WC02999, WC03501, and WC03255, respectively. Notice that total debt consists of short-term debt, current portion of long-term debt, and long-term debt.
} 


\section{Table 1. Descriptive Statistics on Daily Stock Returns of Twenty-Eight U.S. Banks}

\begin{tabular}{|c|c|c|c|c|c|c|}
\hline Banks & Mean & $\begin{array}{l}\text { Std. } \\
\text { Dev. }\end{array}$ & Min. & Max. & Skew. & Kurtosis \\
\hline BancorpSouth & 0.040 & 2.28 & -15.12 & 19.71 & 0.23 & 8.63 \\
\hline Bank of America & 0.031 & 2.70 & -34.12 & 30.21 & -0.35 & 32.80 \\
\hline Bank of Hawaii & 0.045 & 1.78 & -25.51 & 12.95 & -0.63 & 19.77 \\
\hline $\mathrm{BB} \& \mathrm{~T}$ & 0.041 & 2.09 & -26.61 & 21.20 & 0.12 & 19.53 \\
\hline Central Pacific & 0.009 & 3.44 & -66.87 & 69.31 & 0.28 & 68.85 \\
\hline Citigroup & 0.020 & 2.98 & -49.47 & 45.63 & -0.50 & 42.92 \\
\hline City National & 0.029 & 2.29 & -18.92 & 20.21 & 0.13 & 11.15 \\
\hline Community Bank $^{\mathrm{a}}$ & 0.041 & 2.30 & -14.22 & 16.55 & 0.37 & 9.11 \\
\hline Comerica & 0.038 & 2.13 & -22.69 & 18.81 & -0.16 & 18.68 \\
\hline Cullen/Frost Bankers & 0.054 & 2.17 & -21.46 & 19.78 & 0.17 & 13.52 \\
\hline First Horizon National & 0.033 & 2.36 & -44.11 & 25.54 & -1.18 & 45.41 \\
\hline FNB & 0.024 & 2.52 & -25.53 & 19.57 & -0.19 & 14.23 \\
\hline JP Morgan & 0.033 & 2.55 & -32.46 & 22.39 & -0.12 & 17.58 \\
\hline KeyCorp & 0.011 & 2.56 & -40.55 & 43.34 & -0.51 & 50.04 \\
\hline M\&T Bank & 0.054 & 1.75 & -17.59 & 22.83 & 0.33 & 24.60 \\
\hline Marshall \& Ilsley & 0.017 & 2.53 & -30.15 & 32.93 & -0.23 & 40.12 \\
\hline Old National Bancorp & 0.019 & 1.74 & -19.47 & 15.84 & 0.31 & 16.55 \\
\hline $\mathrm{PNC}$ & 0.031 & 2.28 & -53.44 & 31.55 & -1.30 & 68.26 \\
\hline Regions Financial & 0.001 & 2.76 & -52.88 & 39.48 & -0.62 & 56.56 \\
\hline Sterling & 0.017 & 2.27 & -21.26 & 19.39 & 0.27 & 12.36 \\
\hline Suntrust Banks & 0.023 & 2.40 & -31.71 & 26.67 & -0.39 & 29.95 \\
\hline Synovus Financial & 0.017 & 2.78 & -30.07 & 24.86 & -0.02 & 17.68 \\
\hline TCF Financial & 0.047 & 2.35 & -17.65 & 23.53 & 0.49 & 13.73 \\
\hline US Bancorp & 0.054 & 2.17 & -20.05 & 25.76 & 0.22 & 18.69 \\
\hline Valley National Bancorp & 0.034 & 2.22 & -17.48 & 21.71 & 0.29 & 11.39 \\
\hline Webster Financial & 0.024 & 2.56 & -23.51 & 31.03 & 0.07 & 20.03 \\
\hline Wells Fargo & 0.060 & 2.35 & -27.21 & 28.34 & 0.66 & 27.47 \\
\hline Wilmington Trust & 0.026 & 2.11 & -23.12 & 27.62 & 0.05 & 20.85 \\
\hline \multicolumn{7}{|c|}{$\begin{array}{l}\text { Notes: The sample period runs from January } 2,1987 \text {, to December } 31,2009 \text { (sample } \\
\text { size } 6,000 \text { ). All values except the skewness and kurtosis are in percentages. The list } \\
\text { consists of all banks that are preliminarily traded on the NYSE during the sample } \\
\text { period. } \\
{ }^{a} \text { Community Bank N.A. (CBNA) is a bank holding company in Upstate New York. Its } \\
\text { predecessor bank was founded in } 1866 \text {, and it is the wholly owned banking subsidiary } \\
\text { of Community Bank System, Inc. (CBSI). }\end{array}$} \\
\hline
\end{tabular}

kurtosis from the normal distribution, which is 3 . This indicates that the daily stock returns may follow heavy-tailed distributions. Moreover, most of the skewness measures are negative or close to 0 . This 
Table 2. Descriptive Statistics on Yearly Size Measures of Twenty-Eight U.S. Banks

\begin{tabular}{|l|r|r|r|r|r|r|}
\hline & \multicolumn{2}{|c|}{ Total Asset } & \multicolumn{2}{c|}{ Total Equity } & \multicolumn{2}{c|}{ Total Debt } \\
\cline { 2 - 7 } Banks & $\mathbf{2 0 0 9}$ & Average & $\mathbf{2 0 0 9}$ & Average & $\mathbf{2 0 0 9}$ & Average \\
\hline BancorpSouth & 13.2 & 6.5 & 1.3 & 0.6 & 1.0 & 0.5 \\
Bank of America & 2223.3 & 648.4 & 193.6 & 51.4 & 764.7 & 199.3 \\
Bank of Hawaii & 12.4 & 11.3 & 0.9 & 0.9 & 1.7 & 2.2 \\
BB\&T & 165.2 & 55.0 & 16.2 & 5.1 & 29.5 & 11.3 \\
Central Pacific & 4.9 & 2.4 & 0.2 & 0.2 & 0.9 & 0.3 \\
Citigroup & 1856.6 & 1145.3 & 152.4 & 73.1 & 561.3 & 346.8 \\
City National & 20.9 & 8.6 & 1.8 & 0.8 & 1.4 & 0.8 \\
Community Bank & 5.4 & 2.3 & 0.6 & 0.2 & 0.9 & 0.3 \\
Comerica & 59.2 & 38.4 & 4.8 & 3.1 & 11.5 & 6.9 \\
Cullen/Frost Bankers & 16.3 & 7.4 & 1.9 & 0.6 & 0.9 & 0.6 \\
First Horizon National & 26.1 & 18.4 & 2.1 & 1.3 & 6.8 & 4.6 \\
FNB & 8.7 & 3.9 & 1.0 & 0.3 & 1.2 & 0.5 \\
JP Morgan & 2032.0 & 655.8 & 157.2 & 45.9 & 625.3 & 173.5 \\
KeyCorp & 92.7 & 69.7 & 7.9 & 5.2 & 13.6 & 16.6 \\
M\&T Bank & 68.9 & 28.5 & 7.0 & 2.7 & 12.7 & 5.5 \\
Marshall \& Ilsley & 56.2 & 26.0 & 5.3 & 2.4 & 7.5 & 5.3 \\
Old National Bancorp & 7.9 & 6.0 & 0.8 & 0.5 & 1.0 & 1.0 \\
PNC & 269.9 & 87.3 & 21.6 & 6.9 & 39.3 & 18.4 \\
Regions Financial & 142.3 & 48.8 & 14.1 & 5.3 & 22.1 & 8.0 \\
Sterling & 2.1 & 1.2 & 0.1 & 0.1 & 0.3 & 0.2 \\
Suntrust Banks & 174.2 & 92.8 & 17.5 & 8.2 & 22.9 & 17.3 \\
Synovus Financial & 32.8 & 14.6 & 1.9 & 1.4 & 2.2 & 1.4 \\
TCF Financial & 17.9 & 9.7 & 1.2 & 0.7 & 4.8 & 2.4 \\
US Bancorp & 281.2 & 109.6 & 24.5 & 9.6 & 63.9 & 26.9 \\
Valley National Bancorp & 14.3 & 6.9 & 1.3 & 0.5 & 3.3 & 1.1 \\
Webster Financial & 17.6 & 9.0 & 1.5 & 0.7 & 2.0 & 2.1 \\
Wells Fargo & 1240.4 & 295.0 & 103.3 & 23.3 & 242.8 & 69.8 \\
Wilmington Trust & 10.9 & 6.9 & 1.0 & 0.6 & 1.0 & 1.2 \\
\hline Notes: The sample period is from & 1987 to December 2009 twenty-three years). The \\
list consists of all banks that are preliminarily traded on & the NYSE during the sample \\
period. The numbers are in millions of & U.S. dollars. & & & \\
\hline
\end{tabular}

indicates that the heavy-tailedness may come from the downside of the distribution, the losses. Hence, our heavy-tailed assumption on the tail of losses is valid for the data set employed.

From the descriptive statistics of the size measures, we observe a large variation on the size measures of the selected banks. The top three largest banks in the list-Bank of America, JP Morgan, and 
Citigroup - are approximately 1,000 times larger than the smallest bank in the list, Sterling Banc, in all aspects. Although the criterion that the selected banks have to be active in the stock market for twenty-three years may result in a sample selection bias, since such banks are more likely to be large banks, the variation of the size measures shows that the constructed banking system contains both large and small banks.

Using the stock returns is a natural choice for our approach in analyzing the systemic importance. The restriction imposed by the methodology of estimating the $L$ function is that the sample size has to be sufficient; see appendix 2. Moreover, since we intend to perform a moving window analysis, the restriction on the length of the time series is further enhanced. Therefore, daily or higher frequency is necessary for a full non-parametric approach. This limits us to using financial market data. Equity returns are the most convenient choice. Other high-frequency indicators such as CDS spreads are also possible. Nevertheless, the CDS data do not go back for a sufficiently long period, which keeps us from performing a moving window analysis. It is also possible to apply the proposed methodology with low-frequency data, such as return on asset from bank balance sheet. In that case, a full non-parametric estimate on the $L$ function is not applicable. Instead, further modeling on the $L$ function should be considered. In this study we intend to illustrate the methodology without modeling the $L$ function. Hence, we stick to the equity return data.

\subsection{Estimation of the Systemic Importance Measures}

By estimating the $L$ function (for details, see appendix 2), we obtain the estimates of the three systemic importance measures (SII, PAO, and VI) across the full sample period, as shown in table 3. We start with the PAO measure proposed by Segoviano and Goodhart (2009). A general observation is that all of the estimates are quite high (above 60 percent). This is in line with our prediction that the PAO measures of all banks in a system are at a relatively high level and do not differ much from each other. Since the PAO measure is directly connected to the VI measure as shown in corollary 1 , a similar feature is observed for the VI measures. In fact, the order of the VI measures follows that of the PAO measures as proved in corollary 1 . To name 
Table 3. Estimated Systemic Importance Measures: Full Sample Analysis

\begin{tabular}{|l|c|c|r|}
\hline Banks & SII & PAO & VI \\
\hline BancorpSouth & 6.72 & $59.44 \%$ & $6.73 \%$ \\
Bank of America & 10.84 & $94.44 \%$ & $10.28 \%$ \\
Bank of Hawaii & 10.44 & $84.44 \%$ & $9.30 \%$ \\
BB\&T & 10.88 & $86.11 \%$ & $9.46 \%$ \\
Central Pacific & 8.68 & $65.00 \%$ & $7.31 \%$ \\
Citigroup & 10.59 & $90.56 \%$ & $9.90 \%$ \\
City National & 9.30 & $76.11 \%$ & $8.46 \%$ \\
Community Bank & 6.53 & $59.44 \%$ & $6.73 \%$ \\
Comerica & 12.02 & $92.78 \%$ & $10.12 \%$ \\
Cullen/Frost Bankers & 8.05 & $73.89 \%$ & $8.23 \%$ \\
First Horizon National & 10.84 & $83.33 \%$ & $9.19 \%$ \\
FNB & 7.41 & $57.78 \%$ & $6.55 \%$ \\
JP Morgan & 9.76 & $86.67 \%$ & $9.52 \%$ \\
KeyCorp & 12.44 & $93.33 \%$ & $10.18 \%$ \\
M\&T Bank & 11.10 & $86.67 \%$ & $9.52 \%$ \\
Marshall \& Ilsley & 11.92 & $93.89 \%$ & $10.23 \%$ \\
Old National Bancorp & 9.36 & $77.78 \%$ & $8.63 \%$ \\
PNC & 10.73 & $86.11 \%$ & $9.46 \%$ \\
Regions Financial & 11.91 & $90.56 \%$ & $9.90 \%$ \\
Sterling & 8.69 & $65.56 \%$ & $7.37 \%$ \\
Suntrust Banks & 12.11 & $92.78 \%$ & $10.12 \%$ \\
Synovus Financial & 10.11 & $83.89 \%$ & $9.24 \%$ \\
TCF Financial & 10.57 & $81.11 \%$ & $8.96 \%$ \\
US Bancorp & 10.32 & $78.89 \%$ & $8.74 \%$ \\
Valley National Bancorp & 7.82 & $65.00 \%$ & $7.31 \%$ \\
Webster Financial & 9.87 & $82.22 \%$ & $9.07 \%$ \\
Wells Fargo & 10.91 & $90.00 \%$ & $9.85 \%$ \\
Wilmington Trust & $83.33 \%$ & $9.19 \%$ \\
\hline Notes: SII is the systemic importance index, defined as the number of expected \\
bank failures given a particular bank fails; see 2 PAO is the probability of having \\
at least one extra bank failure when a particular bank fails, defined in $(1)$. VI is the \\
vulnerability index, defined as the probability of failure given there exists at least \\
another bank failure in the system; see $(3)$. & & \\
\hline
\end{tabular}

a few banks with the highest PAO and VI measures, Bank of America reports the highest PAO at 94.44 percent, followed by Marshall \& Ilsley with 93.89 percent and KeyCorp with 93.33 percent. The 
corresponding VI measures are 10.28 percent, 10.23 percent, and 10.18 percent, respectively. At the bottom of the list ranked by the PAO measure, we have FNB, BancorpSouth, and Community Bank. ${ }^{5}$

The SII measure introduced in this paper gives a somewhat different outlook compared with the PAO measure. The three lowest SII banks are the same as those with the lowest three PAO measures, although with a different order. The lowest SII measure comes from Community Bank, which is 6.53. This means that if Community Bank is experiencing a crisis, it will be accompanied by an average of 5.53 extra failures in this system. Compared with the size of the system, twenty-eight banks, this is not a high systemic impact. The highest estimated SII measure is 12.44 from KeyCorp, which is almost twice as high as the lowest value. A crisis of KeyCorp will be accompanied by an average of 11.44 extra crises in this system, twice the systemic impact of Community Bank. Hence, we observe a variation of the SII measure across different banks. To name a few with the highest SII measures, the top three are KeyCorp (12.44), Suntrust Banks (12.11), and Comerica (12.02). They are different from the banks with the top-three highest PAO. In general, ranking the PAO measures is different from ranking the SII measures. For example, the bank with the highest PAO, Bank of America, is only ranked at tenth place among all banks when considering the SII measure.

To summarize, the comparison between the three measures shows that although they have different economic backgrounds, the PAO measure and the VI measure are equally informative in terms of ranking the systemic importance of financial institutions. The SII measure, in contrast, provides information on the size of the systemic impact corresponding to the failure of a particular bank. It therefore provides a different view than the other two. Across different banks, the SII measures vary while the PAO measures remain at a high, comparable level. This agrees with our theoretical prediction. Therefore, the SII measure is more informative in distinguishing the systemic importance of financial institutions.

\footnotetext{
${ }^{5}$ Community Bank N.A. (CBNA) is a bank holding company in Upstate New York. Its predecessor bank was founded in 1866, and it is the wholly owned banking subsidiary of Community Bank System, Inc. (CBSI).
} 
Besides estimating the three systemic importance measures from the full sample period, we consider sub-samples for the estimation and perform a moving window approach. By moving the sub-sample window, we could obtain time-varying estimation on the systemic importance measures. We consider the estimation window as 2,000 days (approximately eight years), and then move the estimation window forward month by month. The first possible window ends at September 1994. In other words, the first estimation considers data ending at September 30, 1994, and going back 2,000 days. From then on, we take the end of each month as the ending day of each estimation window and use the data going back 2,000 days. By moving the estimation window month by month, we observe the estimates at the end of each month from September 1994 to December 2009. For simplicity's sake, we only plot the results for selected banks, ${ }^{6}$ as shown in figure 1. The upper panel shows the moving window SII measures, and the bottom panel shows the moving window PAO measures. The two vertical lines in the two figures correspond to the failures of two large investment banks: Bear Stearns (March 2008) and Lehman Brothers (September 2008).

From the moving window SII estimates, we observe that the SII measures gradually increased from 1998 to 2003, then remained relatively stable until the end of 2006. From 2007, there was a sharp rise. The sharp rise of SII started before the failure of Bear Stearns and continued with the failure of Lehman Brothers, until early 2009. From mid-2009, the SII measures became stable and with a slight downward slope. In contrast, the PAO measures are stable across time, particularly for the large banks. Only for the least systemically important bank can some variation be observed. This is due to the fact that the PAO measures of large banks were already at a high level in the early period of our sample. It would thus be difficult to obtain a further rise to a higher level.

The observations from the moving window approach again confirm our theoretical prediction that the PAO measures always stay at a high, comparable level, while the SII measure varies across time

\footnotetext{
${ }^{6}$ We select the least systemically important bank, Community Bank, and the two largest banks, Bank of America and JP Morgan, in the plots. According to the full sample analysis, Bank of America is the most systemically important bank in terms of PAO.
} 


\section{Figure 1. Moving Window Results on Systemic Importance Measures}
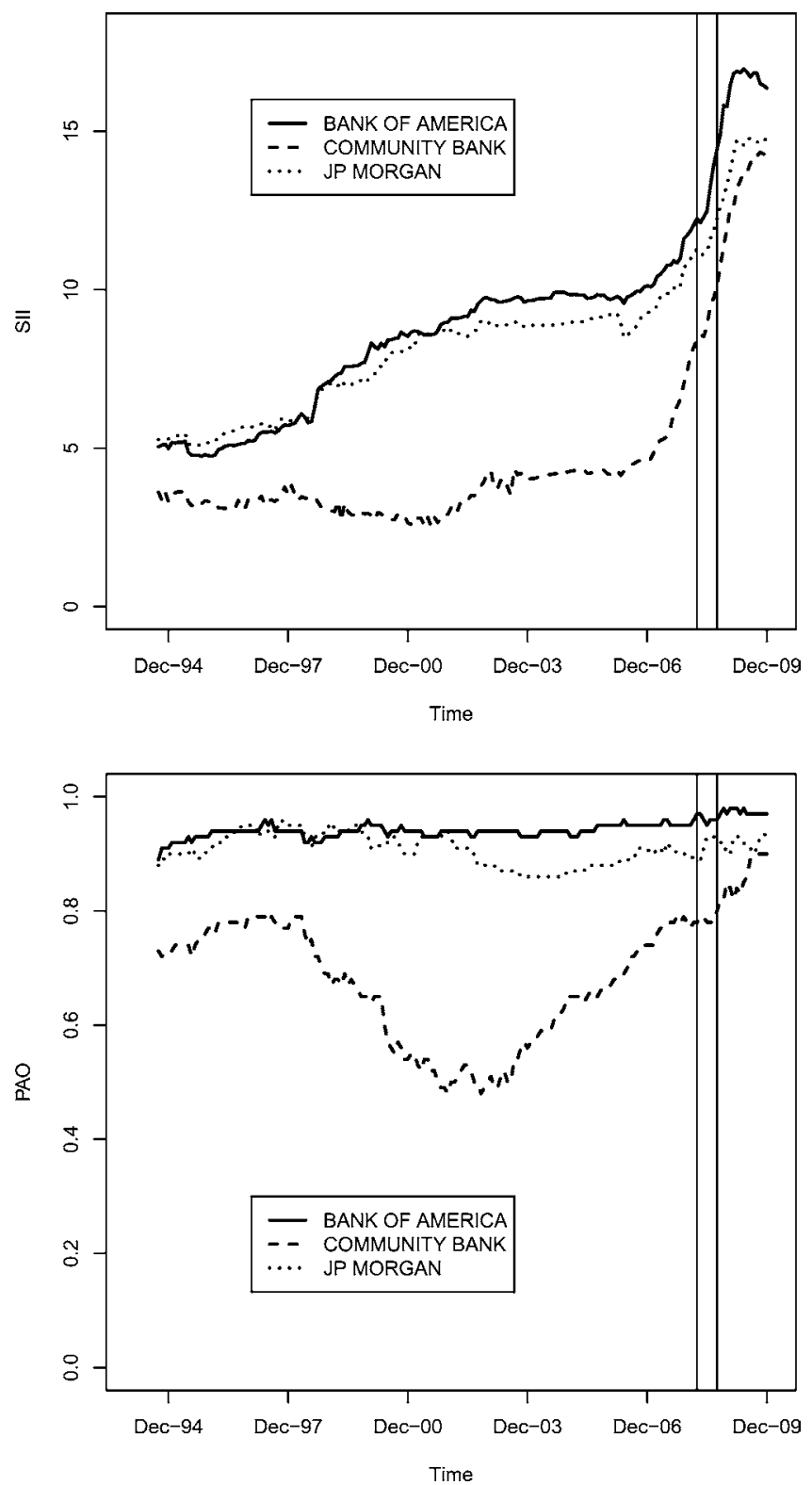

Notes: The moving window measures are estimated from a 2,000-day sub-sample ending at the end of each month. The upper panel presents the results for SII, which is the systemic importance index, defined as the number of expected bank failures given a particular bank fails; see (2). The bottom panel presents the results for PAO, which is the probability of having at least one extra bank failure when a particular bank fails, defined in (1). The two vertical lines refer to the failures of Bear Stearns (March 2008) and Lehman Brothers (September 2008). 
and across institutions. The sharp rise of the SII measures addresses the crisis starting from 2008 and hence is more informative in analyzing the systemic risk in a financial system. Although we observe an early rise before the crisis, we do not emphasize that the SII measure is a predictor of the crisis. The sharp rise of SII measures might either be a predictor of the crisis or an ex post consequence caused by the crisis. The intuition for the latter possibility is as follows. Banks tend to take similar strategies, such as fire sales, during a crisis, which may result in more similar portfolio holdings across all banks. According to the theoretical model in section 4, that similarity leads to an increase of the SII measures on all banks. Hence, the timing of the sharp rising of the SII measures is still an open issue for further study.

There are a few other observations from the moving window analysis. Notice that the financial system we have constructed contains twenty-eight banks. An SII measure of 15 means that if a certain bank fails, there will be an average of fourteen extra bank failures simultaneously. This is half of the entire system, which must be considered as a severe risk. Hence, the observed SII measures from the end of 2008 to mid-2009 indicate that the banking system suffers severe systemic risk during the crisis. Moreover, it is remarkable that Community Bank, the least systemically important bank from the full sample analysis, also showed the least systemic importance during the crisis. Nevertheless, the absolute level of the SII measure reached a comparable level with the other large banks. This suggests that size may not be a good proxy of systemic importance, particularly during periods of crisis.

\subsection{Test "Too Big to Fail"}

We use the estimated systemic importance measures to check whether they are correlated with the size measures. The correlation test is across different banks; thus, we need to have a unified value for each individual bank on each size measure. Since the sample period ends in year 2009, we first consider the end-of-2009 values of each size measure. The second approach is to take an average of the size measures over the full sample period (from 1987 to 2009). Then, we calculate the Pearson correlation coefficients between each pair of size measure and systemic importance measure across 
Table 4. Correlation Coefficients: Full Sample Analysis

\begin{tabular}{|cc|c|c|c|}
\hline & & SII & PAO & VI \\
\hline \multirow{4}{*}{ End of 2009 } & Total Asset & 0.1790 & $0.3968^{* *}$ & $0.3943^{* *}$ \\
& & $(0.3622)$ & $(0.0366)$ & $(0.0379)$ \\
& & 0.1892 & $0.4053^{* *}$ & $0.4027^{* *}$ \\
& Total Equity & $(0.3348)$ & $(0.0324)$ & $(0.0336)$ \\
& & 0.1399 & $0.3640^{*}$ & $0.3615^{*}$ \\
& & $(0.4777)$ & $(0.0569)$ & $(0.0588)$ \\
\hline \multirow{4}{*}{ Average } & Total Asset & 0.1733 & $0.3746^{* *}$ & $0.3723^{*}$ \\
& & $(0.3779)$ & $(0.0495)$ & $(0.0510)$ \\
& Total Equity & 0.1980 & $0.4031^{* *}$ & $0.4006^{* *}$ \\
& & $(0.3126)$ & $(0.0334)$ & $(0.0347)$ \\
& Total Debt & 0.1542 & $0.3546^{*}$ & $0.3523^{*}$ \\
& $(0.4334)$ & $(0.0641)$ & $(0.0660)$ \\
\hline
\end{tabular}

Notes: SII is the systemic importance index, defined as the number of expected bank failures given a particular bank fails; see (2). PAO is the probability of having at least one extra bank failure when a particular bank fails, defined in (1). VI is the vulnerability index, defined as the probability of failure given there exists at least another bank failure in the system; see (3). The numbers in parentheses are the $p$-values for testing whether the correlation coefficient is significantly different from zero. The upper panel reports the results based on using end-of-2009 values of the size measure, while the lower panel reports the results based on using the average of the size measure across the full sample period (from 1987 to 2009). ${ }^{* *}{ }^{*}{ }^{* *}$, and ${ }^{*}$ denote significance at the 1 percent, 5 percent, and 10 percent level, respectively.

twenty-eight banks. Moreover, we test whether the correlation coefficient is significantly different from zero. The results are shown in table 4.

Generally, the PAO and VI measures are positively correlated with the size measures, with significance level 5 percent or 10 percent. Put differently, the SII measure is not correlated with any size measure. We have argued throughout the paper that the SII measure is a more informative measure of systemic importance, because it considers the systemic impact of the failure of a particular bank. We conclude that the systemic impact of a bank failure is not correlated with the size measures. Therefore, "too big to fail" is not valid on the impact level at least for the constructed banking system. Nevertheless, with the positive correlation between the other two 
systemic importance measures and the size measures, the big banks are more likely to cause extra crises in the system. The bottom line is that it is not proper to use the size measures as a proxy of systemic importance. Instead, it is necessary to consider all of the systemic importance measures when identifying systemically important financial institutions.

We have carried out extensive robustness checks for the observed results. First of all, with the full sample estimation on the systemic risk measure, we consider the size measure in other years (e.g., end of 2008,2007 , etc.). The results are comparable with those using the end-of-2009 value. We omit the details.

Secondly, instead of the Pearson correlation, we consider the Spearman correlation, which only emphasizes the correlation between ranking orders. The Spearman correlation coefficients between the SII measure and the size measures are significantly different from zero. Since the Pearson correlation considers the absolute level while the Spearman correlation considers only the ranking orders, we find support of the "too big to fail" argument within the constructed system, only in terms of ranking the order.

Thirdly, with the moving window results on the systemic importance measures, we can get the end-of-year estimates on the systemic importance measures from 1994 to 2009 (sixteen years). We pool all of the bank-year estimates together, which results in $28 \cdot 16=448$ estimates for each systemic importance measure, and also 448 observations for each size measure. We then calculate the Pearson correlation coefficient for each pair and repeat the test on the significancy. The results appear in the first panel of table 5 . None of the three systemic importance measures are correlated with any of the size measures.

Moreover, since we have obtained sixteen-year data on systemic importance and size for twenty-eight banks, we also perform the Pearson correlation analysis at the level of each year. We observe that the significant positive correlation between size and the PAO measure (and the VI measure) is robust for the sub-period 1994-99. From 2000 to 2009, the significance disappears. Interestingly, for the first period, the SII measure is also positively correlated with the three size measures. The (in)significant results are robust within each sub-period. To further explore these phenomena, we divide the period 1994-2009 into two sub-periods: 1994-99 and 2000-09, 


\section{Table 5. Correlation Coefficients: Moving Window Analysis}

\begin{tabular}{|c|c|c|c|c|}
\hline & & SII & PAO & VI \\
\hline Full Sample & $\begin{array}{l}\text { Total Asset } \\
\text { Total Equity } \\
\text { Total Debt }\end{array}$ & $\begin{array}{c}0.0263 \\
(0.8943) \\
0.0474 \\
(0.8107) \\
-0.0080 \\
(0.9678)\end{array}$ & $\begin{array}{c}0.2073 \\
(0.2900) \\
0.2188 \\
(0.2633) \\
0.1665 \\
(0.3971)\end{array}$ & $\begin{array}{c}0.2069 \\
0.2908 \\
0.2183 \\
(0.2645) \\
0.1667 \\
(0.3966)\end{array}$ \\
\hline $\begin{array}{l}\text { Period 1: } \\
\text { 1994-99 }\end{array}$ & $\begin{array}{l}\text { Total Asset } \\
\text { Total Equity } \\
\text { Total Debt }\end{array}$ & $\begin{array}{c}0.4541^{* *} \\
(0.0152) \\
0.4727^{* *} \\
(0.0111) \\
0.4648^{* *} \\
(0.0127)\end{array}$ & $\begin{array}{c}0.4950^{* * *} \\
(0.0074) \\
0.5111^{* * *} \\
(0.0054) \\
0.5014^{* * *} \\
(0.0066)\end{array}$ & $\begin{array}{c}0.4911^{* * *} \\
(0.0080) \\
0.5072^{* * *} \\
(0.0059) \\
0.4973^{* * *} \\
(0.0071)\end{array}$ \\
\hline $\begin{array}{l}\text { Period 2: } \\
2000-09\end{array}$ & $\begin{array}{l}\text { Total Asset } \\
\text { Total Equity } \\
\text { Total Debt }\end{array}$ & $\begin{array}{c}0.2192 \\
(0.2624) \\
0.2261 \\
(0.2473) \\
0.1915 \\
(0.3290)\end{array}$ & $\begin{array}{c}0.2295 \\
(0.2402) \\
0.2374 \\
(0.2238) \\
0.1907 \\
(0.3310)\end{array}$ & $\begin{array}{c}0.2297 \\
(0.2396) \\
0.2375 \\
(0.2235) \\
0.1914 \\
(0.3293)\end{array}$ \\
\hline \multicolumn{5}{|c|}{$\begin{array}{l}\text { Notes: SII is the systemic importance index, defined as the number of expected } \\
\text { bank failures given a particular bank fails; see }(2) \text {. PAO is the probability of hav- } \\
\text { ing at least one extra bank failure when a particular bank fails, defined in }(1) \text {. VI } \\
\text { is the vulnerability index, defined as the probability of failure given there exists at } \\
\text { least another bank failure in the system; see }(3) \text {. The numbers in parentheses are the } \\
\text {-values for testing whether the correlation coefficient is significantly different from } \\
\text { zero. The upper panel reports the results based on pooling all bank-year observations } \\
\text { ( } 448 \text { observations). The middle and lower panels report the results based on pooling } \\
\text { bank-year observations in two periods: } 1994-2000 \text { and } 2001-09 .{ }^{* * *},{ }^{* *} \text {, and }{ }^{*} \text { denote } \\
\text { significance at the } 1 \text { percent, } 5 \text { percent, and } 10 \text { percent level, respectively. }\end{array}$} \\
\hline
\end{tabular}

according to the individual year results. Then we pool bank-year data in each sub-period and repeat the Pearson correlation analysis. The results are reported in the second and third panels of table 5 . It confirms the results from the individual-year analysis: in the first period, all three systemic importance measures are highly correlated with the size measures, while in the second period, the correlations 
disappear. It suggests that using size as a proxy for systemic importance was proper in the 1990s, but that the situation has changed from the beginning of the new century. Therefore, it is particularly important to consider the measures on systemic importance within the current financial world.

Last but not least, using daily equity returns may present a potential problem due to the heteroskedasticity in high-frequency financial returns. Diebold, Schuermann, and Stroughair (2000) argue that the fundamental assumption of EVT - that the observations are independent and identically distributed (i.i.d.) - is usually violated when dealing with high-frequency financial returns. They provide two potential solutions: firstly, one may investigate low-frequency block maxima, which reduces the dependency across time; secondly, one may apply a conditional extreme value model. In line with the second type of solution, Chavez-Demoulin, Davison, and McNeil (2005) consider a point process approach to decluster the financial return data. Notice that the non-i.i.d. problem is mainly for evaluating the univariate tail events. Because our study is under a multivariate framework, which models cross-sectional dependence of tail events, the non-i.i.d. problem on the time dimension is of less concern. Nevertheless, we conduct a robustness check in line with the first type of solution proposed by Diebold, Schuermann, and Stroughair (2000). Instead of daily returns, we consider monthly returns. Similar to the block maxima approach, considering monthly returns reduces the interdependent problem on the time dimension. Due to the low frequency, we have to use the full sample (276 months) for the analysis. The results appear in table 6 . The estimates of the three systemic risk measures are similar to those using daily returns - at least in terms of ranking the systemic importance. The absolute level is higher than that using daily returns, because it is more likely to observe simultaneous tail events in the same month. We repeat the Pearson correlation analysis as shown in table 7 . Again, we confirm that the systemic importance measures are not correlated with the size measures.

\section{Conclusion}

This paper considers three measures of systemic importance of financial institutions in a financial system. Since we regard the system as 
Table 6. Systemic Importance Measures: Monthly Data

\begin{tabular}{|l|r|r|r|}
\hline Banks & \multicolumn{1}{|c|}{ SII } & \multicolumn{1}{c|}{ PAO } & VI \\
\hline BancorpSouth & 10.15 & $75.00 \%$ & $11.28 \%$ \\
Bank of America & 11.70 & $85.00 \%$ & $12.59 \%$ \\
Bank of Hawaii & 11.60 & $100.00 \%$ & $14.49 \%$ \\
BB\&T & 13.50 & $90.00 \%$ & $13.24 \%$ \\
Central Pacific & 8.65 & $90.00 \%$ & $13.24 \%$ \\
Citigroup & 12.00 & $95.00 \%$ & $13.87 \%$ \\
City National & 10.20 & $80.00 \%$ & $11.94 \%$ \\
Community Bank & 9.10 & $75.00 \%$ & $11.28 \%$ \\
Comerica & 13.40 & $100.00 \%$ & $14.49 \%$ \\
Cullen/Frost Bankers & 10.20 & $95.00 \%$ & $13.87 \%$ \\
First Horizon National & 9.85 & $75.00 \%$ & $11.28 \%$ \\
FNB & 9.45 & $75.00 \%$ & $11.28 \%$ \\
JP Morgan & 10.80 & $90.00 \%$ & $13.24 \%$ \\
KeyCorp & 12.45 & $90.00 \%$ & $13.24 \%$ \\
M\&T Bank & 13.10 & $95.00 \%$ & $13.87 \%$ \\
Marshall \& Ilsley & 13.15 & $100.00 \%$ & $14.49 \%$ \\
Old National Bancorp & 8.25 & $70.00 \%$ & $10.61 \%$ \\
PNC & 12.20 & $95.00 \%$ & $13.87 \%$ \\
Regions Financial & 11.85 & $95.00 \%$ & $13.87 \%$ \\
Sterling & 9.80 & $65.00 \%$ & $9.92 \%$ \\
Suntrust Banks & 12.85 & $100.00 \%$ & $14.49 \%$ \\
Synovus Financial & 11.25 & $95.00 \%$ & $13.87 \%$ \\
TCF Financial & 10.60 & $90.00 \%$ & $13.24 \%$ \\
US Bancorp & 12.80 & $100.00 \%$ & $14.49 \%$ \\
Valley National Bancorp & 10.80 & $85.00 \%$ & $12.59 \%$ \\
Webster Financial & 11.35 & $90.00 \%$ & $13.24 \%$ \\
Wells Fargo & 13.50 & $90.00 \%$ & $13.24 \%$ \\
Wilmington Trust & 11.35 & $95.00 \%$ & $13.87 \%$ \\
\hline Notes: SII is the systemic importance index, defined as the number of expected \\
bank failures given a particular bank fails; see 2$).$ PAO is the probability of having \\
at least one extra bank failure when a particular bank fails, defined in $(1)$. VI is the \\
vulnerability index, defined as the probability of failure given there exists at least \\
another bank failure in the system; see $(3)$. & & \\
\hline
\end{tabular}

the combination of individual institutions, it is a multivariate, rather than bilateral, relation. We consider the PAO measure proposed by Segoviano and Goodhart (2009), as well as two new measures: the SII measure, which measures the size of the systemic impact if one 
Table 7. Correlation Coefficients: Monthly Data

\begin{tabular}{|c|c|c|c|c|}
\hline & & SII & PAO & VI \\
\hline End of 2009 & $\begin{array}{l}\text { Total Asset } \\
\text { Total Equity } \\
\text { Total Debt }\end{array}$ & $\begin{array}{c}0.2137 \\
(0.2749) \\
0.2254 \\
(0.2488) \\
0.1489 \\
(0.4497)\end{array}$ & $\begin{array}{c}0.1028 \\
(0.6027) \\
0.1034 \\
(0.6005) \\
0.0673 \\
(0.7335)\end{array}$ & $\begin{array}{c}0.1068 \\
(0.5884) \\
0.1075 \\
(0.5862) \\
0.0714 \\
(0.7182)\end{array}$ \\
\hline Average & $\begin{array}{l}\text { Total Asset } \\
\text { Total Equity } \\
\text { Total Debt }\end{array}$ & $\begin{array}{c}0.1981 \\
(0.3121) \\
0.2259 \\
(0.2478) \\
0.1728 \\
(0.3792) \\
\end{array}$ & $\begin{array}{c}0.1460 \\
(0.4585) \\
0.1530 \\
(0.4371) \\
0.1272 \\
(0.5189) \\
\end{array}$ & $\begin{array}{c}0.1488 \\
(0.4498) \\
0.1560 \\
(0.4279) \\
0.1300 \\
(0.5098)\end{array}$ \\
\hline \multicolumn{5}{|c|}{$\begin{array}{l}\text { Notes: SII is the systemic importance index, defined as the number of expected } \\
\text { bank failures given a particular bank fails; see (2). PAO is the probability of hav- } \\
\text { ing at least one extra bank failure when a particular bank fails, defined in (1). VI } \\
\text { is the vulnerability index, defined as the probability of failure given there exists at } \\
\text { least another bank failure in the system; see (3). The numbers in parentheses are the } \\
p \text {-values for testing whether the correlation coefficient is significantly different from } \\
\text { zero. The upper panel reports the results based on using end-of-2009 values of the } \\
\text { size measure, while the lower panel reports the results based on using the average } \\
\text { of the size measure across the full sample period (from } 1987 \text { to 2009). There is no } \\
\text { significance result under the } 10 \text { percent level. }\end{array}$} \\
\hline
\end{tabular}

bank fails, and the VI measure, which measures the impact on a particular bank when the other part of the system is in distress.

We use a theoretical model based on affine portfolio holdings to show that a large bank is not necessarily more systemically important in terms of the three proposed systemic importance measures. Only with diversified banking activities might a large bank become systemically important. In contrast, the crisis of an isolated large bank will not pose a threat to the system.

The discussion can be extended to regulation policy debate. With acknowledgment of the trade-off between micro-level risk management and systemic importance, we must conclude that there is a great need for macroprudential approaches on financial regulation and supervision. Moreover, measuring systemic importance is the 
key to identifying systemically important institutions when imposing macroprudential regulations.

Besides developing the theoretical model, we conduct an empirical analysis - using multivariate EVT - to estimate the systemic importance measures. The empirical observation confirms that the PAO measure is not as informative as the SII measure in terms of distinguishing the systemically important banks. A moving window analysis shows similar results. Moreover, the VI measure is shown to be as informative as the PAO measure in terms of identifying systemically important banks.

We use the estimated systemic importance measures to test whether they are correlated with the measures on bank size. Regarding the systemic impact of bank failure measured by the SII measure, there is no empirical evidence supporting the "too big to fail" argument in terms of the Pearson correlation. In contrast, the other two systemic importance measures, PAO and VI, are positively correlated to the size measures. When considering the Spearman correlation, we find support for "too big to fail." Moreover, we find that in the more recent period the correlations disappear, which suggests that particular attention should be given to the systemic importance measures in recent years.

The empirical analysis in this paper is based on an artificial bank system. Therefore, the evidence from the empirical analysis should not be regarded as either support or disproof of the "too big to fail" argument. The bottom line is that we show the possibility of having a banking system in which the size measures are not a good proxy of the systemic importance.

Although in the current empirical analysis our proposed SII measure is shown to be more informative than the PAO measure proposed by Segoviano and Goodhart (2009), we address one potential drawback of the SII measure: it is a simple counting measure that takes no account of the differences between potential losses when different financial institutions fail. In other words, when calculating the expected number of failures in the system, the SII measure does not distinguish whether it causes a failure of a big bank or a small bank. This could be improved by considering the expected total loss in the system if one bank fails - i.e., calculating the expected shortfall conditional on a certain bank failure, which incorporates the size of all banks. Acharya, Santos, and Yorulmazer (2010) have 
designed systemic importance measures in this manner, while taking the heavy-tailedness of individual returns into consideration. To model the dependence structure, they use dynamic conditional correlation (DCC) models, which have an EVT flavor but deviate from the multivariate EVT framework. A systemic importance measure addressing the conditional expected shortfall under the multivariate EVT framework may overcome the drawback of the proposed SII measure. This is left for future research.

\section{Appendix 1. Proofs}

\section{Proof of Proposition 1}

Recall the definition of the PAO measure in (1). We have that

$$
\begin{aligned}
P A O_{i}(p)= & \frac{P\left(\left\{\exists j \neq i, \text { s.t. } X_{j}>\operatorname{VaR}(p)\right\} \cap\left\{X_{i}>\operatorname{VaR}(p)\right\}\right)}{P\left(X_{i}>\operatorname{VaR}(p)\right)} \\
= & \frac{1}{p} P\left(\left\{\exists j \neq i, \quad \text { s.t. } X_{j}>\operatorname{VaR}(p)\right\}\right)+1 \\
& -\frac{1}{p} P\left(\left\{\exists j \neq i \text {, s.t. } X_{j}>\operatorname{Va} R_{j}(p)\right\} \cup\left\{X_{i}>\operatorname{VaR}(p)\right\}\right) \\
= & \frac{1}{p} P\left(\left\{\exists j \neq i \text {, s.t. } X_{j}>\operatorname{VaR}(p)\right\}\right)+1 \\
& -\frac{1}{p} P\left(\left\{\exists j, \text { s.t. } X_{j}>\operatorname{VaR}(p)\right\}\right) \\
= & : I_{1}+1-I_{2} .
\end{aligned}
$$

From the definition of the $L$ function in (4), as $p \rightarrow 0, I_{1} \rightarrow$ $L_{\neq i}(1,1, \ldots, 1)$ and $I_{2} \rightarrow L(1,1, \ldots, 1)$, which implies (6).

Proof of Proposition 3

Recall the definition of the SII measure in (2). We have that

$$
\operatorname{SII}_{i}(p)=\sum_{j=1}^{d} E\left(1_{X_{j}>\operatorname{VaR}(p)} \mid X_{i}>\operatorname{VaR}(p)\right)
$$




$$
\begin{aligned}
= & \sum_{j=1}^{d} P\left(X_{j}>\operatorname{VaR}_{j}(p) \mid X_{i}>\operatorname{VaR}_{i}(p)\right) \\
= & \sum_{j=1}^{d} \frac{P\left(X_{j}>\operatorname{Va} R_{j}(p), X_{i}>\operatorname{VaR}_{i}(p)\right)}{P\left(X_{i}>\operatorname{VaR}(p)\right)} \\
= & \sum_{j=1}^{d} \frac{P\left(X_{j}>\operatorname{VaR}(p)\right)+P\left(X_{i}>\operatorname{VaR}(p)\right)}{p\left(X_{j}>V a R_{j}(p) \text { or } X_{i}>\operatorname{VaR}(p)\right)} \\
= & \sum_{j=1}^{d} 2-\frac{P\left(X_{j}>\operatorname{VaR}(p) \text { or } X_{i}>\operatorname{VaR}(p)\right)}{p} .
\end{aligned}
$$

From the definition of the $L$ function in (4), as $p \rightarrow 0$,

$$
\frac{P\left(X_{j}>V_{a} R_{j}(p) \text { or } X_{i}>V a R_{i}(p)\right)}{p} \rightarrow L_{i, j}(1,1) .
$$

The relation (8) is thus proved.

Proof of Corrollary 1

Since $L(1,1, \ldots, 1)-1<0$, the relation (7) implies that a higher value of the VI measure corresponds to a higher level of $L_{\neq i}(1,1, \ldots, 1)$. Together with (6), the corollary follows.

Proof of Theorem 1

Firstly, since the heavy-tailed feature in (11) assumes that the right tail of $Y_{i}$ dominates its left tail, it is sufficient to assume that $Y_{i}$ are all positive random variables for $i=1,2,3$, i.e., without the left tail. We adopt this assumption in the rest of the proof.

We use the Feller convolution theorem to deal with the sum of independently heavy-tailed distributed random variables as in the following lemma.

Lemma 2. Suppose positive random variables $U$ and $V$ are independent. Assume that they are both heavy-tailed distributed with the same tail index $\alpha$. Then, as $s \rightarrow \infty$, 


$$
P(U+V>s) \sim P(U>s)+P(V>s)
$$

Notice that the heavy-tailed feature implies $P(U>s) P(V>$ $s)=o(P(U>s)+P(V>s))$, as $s \rightarrow \infty$. Hence, the Feller convolution theorem is equivalent to

$$
P(U+V>s) \sim P(\max (U, V)>s)
$$

i.e., the sum and the maximum of two independently heavy-tailed distributed random variables are tail equivalent. A proof using sets manipulation can be found in Embrechts, Küppelberg, and Mikosch (1997). With an analogous proof under multivariate framework, a multivariate version of the Feller theorem can be obtained. In the multivariate case, the tail equivalence between two random vectors is defined as the combination of having tail equivalence for each marginal distribution and having the same $L$ function for the tail dependence structure. We present the result in a two-dimensional context in the following lemma without providing the proof.

LEMMA 3. Suppose positive random variables $U_{1}$ and $U_{2}$ are independent. Assume that they are both heavy-tailed distributed with the same tail index $\alpha$. Then for any positive constants $m_{i j}, 1 \leq i, j \leq 2$, we have that the distribution functions of $\left(m_{11} U_{1}+m_{12} U_{2}, m_{21} U_{1}+\right.$ $\left.m_{22} U_{2}\right)$ and $\left(m_{11} U_{1} \vee m_{12} U_{2}, m_{21} U_{1} \vee m_{22} U_{2}\right)$ are tail equivalent.

To prove theorem 1 , it is necessary to calculate the $L$ function of $\left(X_{1}, X_{2}, X_{3}\right)$ on points $(1,1,0),(1,0,1)$, and $(0,1,1)$. The main instrument in the calculation is lemma 3 . We start by comparing the individual risks taken by the three banks.

Proposition 4. For the three-bank model on $\left(X_{1}, X_{2}, X_{3}\right)$, as $p \rightarrow 0$,

$$
\operatorname{VaR}_{2}(p)=\operatorname{VaR}_{3}(p) \sim c V a R_{1}(p)
$$

where

$$
c:=\left(\frac{(2-2 \gamma)^{\alpha}+2 \gamma^{\alpha}}{\gamma^{\alpha}+\mu^{\alpha}+(1-\gamma-\mu)^{\alpha}}\right)^{-1 / \alpha}
$$




\section{Proof of Proposition 4}

From lemma 2, we get that, as $s \rightarrow \infty$,

$$
\begin{aligned}
P\left(X_{1}>s\right) & \sim P\left((2-2 \gamma) Y_{1}>s\right)+P\left(\gamma Y_{2}>s\right)+P\left(\gamma Y_{3}>s\right) \\
& =\left((2-2 \gamma)^{\alpha}+2 \gamma^{\alpha}\right) s^{-\alpha} K(s) .
\end{aligned}
$$

Similarly, we get that

$$
P\left(X_{2}>s\right)=P\left(X_{3}>s\right) \sim\left(\gamma^{\alpha}+\mu^{\alpha}+(1-\gamma-\mu)^{\alpha}\right) s^{-\alpha} K(s) .
$$

By comparing (14) and (15), the relation (13) is a direct consequence.

We remark that $c<1$. This is in line with the fact that the large bank $X_{1}$ takes more risks than the small banks. Moreover, when $\gamma>1 / 2$, we get $c>1 / 2$. In this case, the comparison between bank risk taking is not proportional to the size. At a relative level, the small banks are taking more risk. In other words, the large bank $X_{1}$ benefits from diversification.

Next, we calculate $L(1,1,0)$. Denote $v(p):=\operatorname{VaR}(p)$. From lemma 3 , we get that, as $p \rightarrow 0$,

$$
\begin{aligned}
P( & \left.X_{1}>V a R_{1}(p) \text { or } X_{2}>V a R_{2}(p)\right) \\
\sim & P\left((2-2 \gamma) Y_{1} \vee \gamma Y_{2} \vee \gamma Y_{3}>v(p)\right. \text { or } \\
& \left.\gamma Y_{1} \vee(1-\gamma-\mu) Y_{2} \vee \mu Y_{3}>c v(p)\right) \\
= & P\left(Y_{1}>\frac{v(p)}{(2-2 \gamma) \vee \frac{\gamma}{c}} \text { or } Y_{2}>\frac{v(p)}{\gamma \vee \frac{1-\gamma-\mu}{c}} \text { or } Y_{3}>\frac{v(p)}{\gamma \vee \frac{\mu}{c}}\right) \\
\sim & P\left(Y_{1}>\frac{v(p)}{(2-2 \gamma) \vee \frac{\gamma}{c}}\right)+P\left(Y_{2}>\frac{v(p)}{\gamma \vee \frac{1-\gamma-\mu}{c}}\right) \\
& +P\left(Y_{3}>\frac{v(p)}{\gamma \vee \frac{\mu}{c}}\right) \\
\sim & {\left[\left((2-2 \gamma) \vee \frac{\gamma}{c}\right)^{\alpha}+\left(\gamma \vee \frac{\mu}{c}\right)^{\alpha}+\left(\gamma \vee \frac{1-\gamma-\mu}{c}\right)^{\alpha}\right] } \\
& \times P\left(Y_{1}>v(p)\right) .
\end{aligned}
$$


From (14), we get that $p \sim\left((2-2 \gamma)^{\alpha}+2 \gamma^{\alpha}\right) P\left(Y_{1}>v(p)\right)$ as $p \rightarrow 0$. Together with (16) and (5), we have that

$$
\begin{aligned}
L(1,1,0) & =\lim _{p \rightarrow 0} \frac{P\left(X_{1}>V_{a} R_{1}(p) \text { or } X_{2}>V a R_{2}(p)\right)}{p} \\
& =\frac{\left((2-2 \gamma) \vee \frac{\gamma}{c}\right)^{\alpha}+\left(\gamma \vee \frac{\mu}{c}\right)^{\alpha}+\left(\gamma \vee \frac{1-\gamma-\mu}{c}\right)^{\alpha}}{(2-2 \gamma)^{\alpha}+2 \gamma^{\alpha}} .
\end{aligned}
$$

Due to the symmetry, we have that $L(1,0,1)=L(1,1,0)$. Following a similar calculation, we obtain that

$$
L(0,1,1)=\frac{\left(\frac{\gamma}{c}\right)^{\alpha}+2\left(\frac{\mu \vee(1-\gamma-\mu)}{c}\right)^{\alpha}}{(2-2 \gamma)^{\alpha}+2 \gamma^{\alpha}} .
$$

Proposition 3 implies that $S I I_{2}=S I I_{3}$. Moreover, $S I I_{1}>S I I_{2}$ if and only if $L(1,1,0)<L(0,1,1)$. Hence it is only necessary to compare the values of the $L$ function at the two points.

Denote

$$
Q:=\left((2-2 \gamma)^{\alpha}+2 \gamma^{\alpha}\right)(L(1,1,0)-L(0,1,1)) .
$$

We study the sign of $Q$ in order to compare the systemic importance measures $S I_{i}, i=1,2,3$.

CASE 1: $\quad \frac{2}{3} \leq \gamma<1$

Since $\gamma>1 / 2$, we have $c>1 / 2$. Thus, $\frac{\gamma}{1-\gamma}>2>\frac{1}{c}$, which implies that

$$
\gamma>\frac{1-\gamma}{c}>\max \left(\frac{1-\gamma-\mu}{c}, \frac{\mu}{c}\right)
$$

Therefore

$$
\begin{aligned}
Q= & \left(\left((2-2 \gamma) \vee \frac{\gamma}{c}\right)^{\alpha}+2 \gamma^{\alpha}\right) \\
& -\left(\left(\frac{\gamma}{c}\right)^{\alpha}+2\left(\frac{\mu \vee(1-\gamma-\mu)}{c}\right)^{\alpha}\right)>0 .
\end{aligned}
$$

Hence, $S I I_{1}<S I I_{2}=S I I_{3}$. 
CASE 2: $\quad \gamma=1 / 2$

In this case, we still have $c \geq 1 / 2$. The equality holds if and only if $\mu=1 / 4$. Due to the symmetric position between $X_{2}$ and $X_{3}$, without loss of generality, we assume that $\mu \leq 1 / 4$. Then we get $\mu \leq 1-\gamma-\mu$. Moreover, the inequality $\mu / \gamma \leq 1 / 2 \leq c$ implies that $\gamma \geq \frac{\mu}{c}$ and it is not difficult to obtain that $\gamma \leq \frac{1-\gamma-\mu}{c}$. Hence,

$$
\begin{aligned}
Q & =\left(1+2^{-\alpha}+\left(\frac{1 / 2-\mu}{c}\right)^{\alpha}\right)-\left(\left(\frac{\gamma}{c}\right)^{\alpha}+2\left(\frac{1 / 2-\mu}{c}\right)^{\alpha}\right) \\
& =1+2^{-\alpha}-\left(\frac{1}{2^{\alpha}}+\left(\frac{1}{2}-\mu\right)^{\alpha}\right) c^{-\alpha} \\
& =1+\frac{1}{2^{\alpha}}-\frac{1+(1-2 \mu)^{\alpha}}{2^{\alpha}} \frac{2^{\alpha}+2}{1+(2 \mu)^{\alpha}+(1-2 \mu)^{\alpha}} \\
& =1+\frac{1}{2^{\alpha}}-\left(1+\frac{2}{2^{\alpha}}\right)\left(1-\frac{(2 \mu)^{\alpha}}{1+(2 \mu)^{\alpha}+(1-2 \mu)^{\alpha}}\right) \\
& \leq 1+\frac{1}{2^{\alpha}}-\left(1+\frac{2}{2^{\alpha}}\right)\left(1-\frac{(1 / 2)^{\alpha}}{1+(1 / 2)^{\alpha}+(1 / 2)^{\alpha}}\right) \\
& =0 .
\end{aligned}
$$

Here we used the facts that $\frac{(2 \mu)^{\alpha}}{1+(2 \mu)^{\alpha}+(1-2 \mu)^{\alpha}}$ is an increasing function with respect to $\mu$ and $\mu \leq 1 / 4$. The equality holds if and only if $\mu=$ $1 / 4$. Hence, in case $\gamma=1 / 2$, we conclude that $S I I_{1} \geq S I I_{2}=S I I_{3}$, with the equality holding if and only if $\mu=1 / 4$.

Case 3: $\quad 0<\gamma \leq 1 / 3$

In this case, it is not difficult to verify that $2-2 \gamma>\frac{\gamma}{c}$ and $\gamma<\frac{1-\gamma}{2 c}$. Due to the symmetry, we only consider the case $0<\mu \leq \frac{1-\gamma}{2}$. Then we have that $\mu \leq 1-\gamma-\mu$ and $\gamma<\frac{1-\gamma-\mu}{c}$. Therefore

$$
Q=(2-2 \gamma)^{\alpha}+\left(\gamma \vee \frac{\mu}{c}\right)^{\alpha}-\frac{\gamma^{\alpha}+(1-\gamma-\mu)^{\alpha}}{c^{\alpha}}
$$

For any fixed $\gamma, c$ is a function of $\mu$ denoted by $c(\mu)$. For $0<\mu<$ $\frac{1-\gamma}{2}, c(\mu)$ is a strictly decreasing function. Thus $g(\mu):=\frac{\mu}{c(\mu)}$ is a continuous, strictly increasing function. Notice that $g(0)=0<\gamma$ and $g((1-\gamma) / 2)>\gamma$. There must exist a unique $\mu^{*} \in(0,(1-\gamma) / 2)$ such that $g\left(\mu^{*}\right)=\gamma$. 
Denote $c^{*}:=c\left(\mu^{*}\right)$. From $\frac{\mu^{*}}{c^{*}}=\gamma$, we get that

$$
\frac{\gamma^{\alpha}}{\mu^{* \alpha}}=\left(c^{*}\right)^{-\alpha}=\frac{(2-2 \gamma)^{\alpha}+2 \gamma^{\alpha}}{\gamma^{\alpha}+\mu^{* \alpha}+\left(1-\gamma-\mu^{*}\right)^{\alpha}} .
$$

It implies that

$$
\frac{\gamma^{\alpha}}{\mu^{* \alpha}}=\left(c^{*}\right)^{-\alpha}=\frac{(2-2 \gamma)^{\alpha}+\gamma^{\alpha}}{\gamma^{\alpha}+\left(1-\gamma-\mu^{*}\right)^{\alpha}} .
$$

Continuing from equation (19), we get that

$$
Q\left(\mu^{*}\right)=(2-2 \gamma)^{\alpha}+\gamma^{\alpha}-\left(\gamma^{\alpha}+\left(1-\gamma-\mu^{*}\right)^{\alpha}\right)\left(c^{*}\right)^{-\alpha}=0 .
$$

Hence, we conclude that for $\mu=\mu^{*}, S I I_{1}=S I I_{2}=S I I_{3}$.

For $0<\mu<\mu^{*}$, it is clear that

$$
\begin{aligned}
Q(\mu) & =(2-2 \gamma)^{\alpha}+\gamma^{\alpha}-\left(\gamma^{\alpha}+(1-\gamma-\mu)^{\alpha}\right) c^{-\alpha} \\
& =(2-2 \gamma)^{\alpha}+\gamma^{\alpha}-\left(\gamma^{\alpha}+(1-\gamma-\mu)^{\alpha}\right) \frac{(2-2 \gamma)^{\alpha}+2 \gamma^{\alpha}}{\gamma^{\alpha}+\mu^{\alpha}+(1-\gamma-\mu)^{\alpha}} \\
& =\frac{\left((2-2 \gamma)^{\alpha}+\gamma^{\alpha}\right) \mu^{\alpha}-\gamma^{\alpha}\left(\gamma^{\alpha}+(1-\gamma-\mu)^{\alpha}\right)}{\gamma^{\alpha}+\mu^{\alpha}+(1-\gamma-\mu)^{\alpha}}
\end{aligned}
$$

is a strictly increasing function with respect to $\mu$. Moreover, for $\mu^{*}<\mu<\frac{1-\gamma}{2}, Q$ is calculated as

$$
\begin{aligned}
Q(\mu) & =(2-2 \gamma)^{\alpha}+\left(\mu^{\alpha}-\gamma^{\alpha}-(1-\gamma-\mu)^{\alpha}\right) c^{-\alpha} \\
& =(2-2 \gamma)^{\alpha}+\left(\mu^{\alpha}-\gamma^{\alpha}-(1-\gamma-\mu)^{\alpha}\right) \frac{(2-2 \gamma)^{\alpha}+2 \gamma^{\alpha}}{\gamma^{\alpha}+\mu^{\alpha}+(1-\gamma-\mu)^{\alpha}} \\
& =\frac{2(2-2 \gamma)^{\alpha} \mu^{\alpha}+2 \gamma^{\alpha}\left(\mu^{\alpha}-\gamma^{\alpha}-(1-\gamma-\mu)^{\alpha}\right)}{\gamma^{\alpha}+\mu^{\alpha}+(1-\gamma-\mu)^{\alpha}},
\end{aligned}
$$

which is also a strictly increasing function with respect to $\mu$. Therefore, for $0<\mu<\mu^{*}, Q<0$ while for $\mu^{*}<\mu<\frac{1-\gamma}{2}, Q>0$. Correspondingly, we have $S I_{1}>S I I_{2}=S I I_{3}$ in the former case and $S I I_{1}<S I_{2}=S I I_{3}$ in the latter case.

Due to the symmetry, a similar result holds when $\frac{1-\gamma}{2}<\mu<$ $1-\gamma$, and the switch point is then $1-\gamma-\mu^{*}$. More specifically, for $\frac{1-\gamma}{2}<\mu<1-\gamma-\mu^{*}, S I_{1}<S I I_{2}=S I I_{3}$; and for $\mu=1-\gamma-\mu^{*}$, $S I_{1}=S I_{2}=S I I_{3}$; for $1-\gamma-\mu^{*}<\mu<1-\gamma, S I_{1}>S I_{2}=S I_{3}$. The theorem is thus proved for case 3 . 


\section{Proof of Lemma 1}

Along the lines of the proof of theorem 1, we obtained that in a three-bank model,

$$
\begin{aligned}
S I I_{1}-S I I_{2}= & \{1+(2-L(1,1,0))+(2-L(1,0,1))\} \\
& -\{(2-L(1,1,0))+1+(2-L(0,1,1))\} \\
= & L(0,1,1)-L(1,0,1) .
\end{aligned}
$$

Therefore, $S I I_{i}>S I I_{j}$ if and only if $L_{\neq i}(1,1, \ldots, 1)>$ $L_{\neq j}(1,1, \ldots, 1)$. Together with $(6)$, the lemma is proved.

We remark that the above calculation is not based on the affine portfolio model. Hence, the result is valid in a general three-bank model. However, one cannot obtain similar relations when the number of banks exceeds three.

\section{Appendix 2. Statistical Estimation on the $L$ Function}

Consider independently and identically distributed (i.i.d.) observations from the random vector $\left(X_{1}, \ldots, X_{d}\right)$ denoted by

$$
\left\{\left(X_{1 s}, X_{2 s}, \ldots, X_{d s}\right) \mid 1 \leq s \leq n\right\}
$$

The sample size is $n$. The non-parametric approach of estimating the $L$ function starts from the assumption (4). Roughly speaking, the estimation takes a certain $p$-value for which the VaR for each dimension can be estimated by the order statistics. Then the probability in the numerator of (4) is estimated by a counting measure. To ensure that $p \rightarrow 0$, theoretically we take a sequence $k:=k(n)$, such that $k(n) \rightarrow \infty$ and $k(n) / n \rightarrow 0$ as $n \rightarrow \infty$, and get an empirical estimation of the $L$ function from replacing $p$ with $k / n$ and using the empirical estimation on the distribution function of $\left(X_{1}, X_{2} \ldots, X_{d}\right)$. The explicit estimator is given as

$$
\hat{L}\left(x_{1}, \ldots, x_{d}\right):=\frac{1}{k} \sum_{s=1}^{n} 1_{\exists 1 \leq i \leq d} \text {, s.t. } X_{i s}>X_{i, n-\left[k x_{i}\right]},
$$


where $X_{i, 1} \leq X_{i, 2} \leq \cdots \leq X_{i, n}$ are the order statistics of the $i$-th dimension of the sample, $X_{i 1}, \ldots, X_{i n}$, for $1 \leq i \leq d$. Particularly, $L(1,1, \ldots, 1)$ is estimated by

$$
\hat{L}(1,1, \ldots, 1):=\frac{1}{k} \sum_{s=1}^{n} 1_{\exists 1 \leq i \leq d, \text { s.t. } X_{i s}>X_{i, n-k}} \text {. }
$$

For the estimator of the $L$ function, usual statistical properties, such as consistency and asymptotic normality, have been proved. See, e.g., de Haan and Ferreira (2006).

Practically, the theoretical conditions on $k$ are not relevant for a finite sample analysis. Thus, how to choose a proper $k$ in the estimator is a major issue in estimation. Instead of taking an arbitrary $k$, a usual procedure is to calculate the estimator of $L(1,1, \ldots, 1)$ under different $k$ values and draw a line plot against the $k$ values. With a low $k$ value, the estimation exhibits a large variance, while for a high $k$ value, since the estimation uses too many observations in the moderate level, it bears a potential bias. Therefore, $k$ is usually chosen by picking the first stable part of the line plot starting from low $k$, which balances the trade-off between the variance and the bias. The estimates follow from the $k$ choice. Because $k$ is chosen from a stable part of the line plot, a small variation of the $k$ value does not change the estimated value. Thus, the exact $k$ value is not sensitive for the estimation on the $L$ function. Such a procedure has been applied in univariate EVT for tail index estimation.

With the chosen $k$, we in fact consider a tail event as the loss return exceeds a $\mathrm{VaR}$ with tail probability level $k / n$. In our empirical application, the chosen $k$ value differs according to the sample size. For the full sample analysis (sample size 6,000), we choose $k=180$, which corresponds to a $p$ level at 3 percent; for the moving window analysis (sample size 2,000), we choose $k=100$, which corresponds to $p=5 \%$; for the monthly data analysis (sample size 276), we choose $k=20$, which corresponds to $p=7.2 \%$. With a lower number of observations, we choose a slightly higher level of $p$, albeit in a low absolute level. 


\section{References}

Acharya, V. V., J. A. C. Santos, and T. Yorulmazer. 2010. "Systemic Risk and Deposit Insurance Premiums." Economic Policy Review (Federal Reserve Bank of New York) 16 (1): 89-98.

Acharya, V. V., and T. Yorulmazer. 2007. "Too Many to FailAn Analysis of Time-Inconsistency in Bank Closure Policies." Journal of Financial Intermediation 16 (1): 1-31.

Adrian, T., and M. Brunnermeier. 2008. "CoVaR." Federal Reserve Bank of New York Staff Report No. 348.

Allen, F., E. Carletti, and A. Babus. 2009. "Financial Crises: Theory and Evidence." Annual Review of Financial Economics 1 (1): $97-116$.

Allen, F., and D. Gale. 2000. "Financial Contagion." Journal of Political Economy 108: 1-33.

Bernanke, B. 2009. "Financial Reform to Address Systemic Risk." Speech at the Council on Foreign Relations, Washington, DC, March 10.

Chavez-Demoulin, V., A. C. Davison, and A. J. McNeil. 2005. "Estimating Value-at-Risk: A Point Process Approach." Quantitative Finance 5 (2): 227-34.

Cifuentes, R., H. S. Shin, and G. Ferrucci. 2005. "Liquidity Risk and Contagion." Journal of the European Economic Association $3(2-3): 556-66$.

Dasgupta, A. 2004. "Financial Contagion through Capital Connections: A Model of the Origin and Spread of Bank Panics." Journal of the European Economic Association 2 (6): 1049-84.

De Bandt, O., and P. Hartmann. 2001. "Systemic Risk in Banking: A Survey." In Financial Crisis, Contagion, and the Lender of Last Resort: A Reader, ed. C. Goodhart and G. Illing, 249-98. Oxford University Press.

De Haan, L., and A. Ferreira. 2006. Extreme Value Theory: An Introduction. Springer.

De Vries, C. G. 2005. "The Simple Economics of Bank Fragility." Journal of Banking and Finance 29 (4): 803-25.

Diebold, F. X., T. Schuermann, and J. D. Stroughair. 2000. "Pitfalls and Opportunities in the Use of Extreme Value Theory in Risk Management." Journal of Risk Finance 1 (2): 30-35. 
Diebold, F. X., and K. Yilmaz. 2009. "Measuring Financial Asset Return and Volatility Spillovers, with Application to Global Equity Markets." Economic Journal 119 (534): 158-71.

Embrechts, P., C. Klüppelberg, and T. Mikosch. 1997. Modelling Extremal Events: for Insurance and Finance. Springer.

Freixas, X., B. M. Parigi, and J.-C. Rochet. 2000. "Systemic Risk, Interbank Relations, and Liquidity Provision by the Central Bank." Journal of Money, Credit, and Banking 32 (3): 611-38. Hartmann, P., S. Straetmans, and C. G. de Vries. 2004. "Asset Market Linkages in Crisis Periods." Review of Economics and Statistics 86 (1): 313-26.

_ 2005. "Banking System Stability: A Cross-Atlantic Perspective." NBER Working Paper No. 11698.

International Monetary Fund. 2009. Global Financial Stability Report (April).

Lagunoff, R., and S. L. Schreft. 2001. "A Model of Financial Fragility." Journal of Economic Theory 99 (1): 220-64.

Poon, S., M. Rockinger, and J. Tawn. 2004. "Extreme Value Dependence in Financial Markets: Diagnostics, Models, and Financial Implications." Review of Financial Studies 17 (2): 581-610.

Rajan, R. G. 2009. "Too Systemic to Fail: Consequences, Causes and Potential Remedies." Written statement to the Senate Banking Committee Hearings, May 6.

Segoviano, M., and C. Goodhart. 2009. "Banking Stability Measures." IMF Working Paper No. 09/04.

Stern, G. H., and R. J. Feldman. 2004. Too Big to Fail: The Hazards of Bank Bailouts. Brookings Institution Press.

Zhou, C. 2008. On Extreme Value Statistics. PhD Thesis, Tinbergen Institute. 